\title{
Improving Data-Analytics Performance Via Autonomic Control of Concurrency and Resource Units
}

\author{
GIL JAE LEE and JOSÉ A. B. FORTES, Electrical and Computer Engineering Department, \\ University of Florida, USA
}

\begin{abstract}
Many big-data processing jobs use data-analytics frameworks such as Apache Hadoop (currently also known as YARN). Such frameworks have tunable configuration parameters set by experienced system administrators and/or job developers. However, tuning parameters manually can be hard and time-consuming because it requires domain-specific knowledge and understanding of complex inter-dependencies among parameters. Most of the frameworks seek efficient resource management by assigning resource units to jobs, the maximum number of units allowed in a system being part of the static configuration of the system. This static resource management has limited effectiveness in coping with job diversity and workload dynamics, even in the case of a single job. The work reported in this article seeks to improve performance (e.g., multiple-jobs makespan and job completion time) without modification of either the framework or the applications and avoiding problems of previous self-tuning approaches based on performance models or resource usage. These problems include (1) the need for time-consuming training, typically offline and (2) unsuitability for multi-jobs/tenant environments. This article proposes a hierarchical self-tuning approach using (1) a fuzzy-logic controller to dynamically adjust the maximum number of concurrent jobs and (2) additional controllers (one for each cluster node) to adjust the maximum number of resource units assigned to jobs on each node. The fuzzylogic controller uses fuzzy rules based on a concave-downward relationship between aggregate CPU usage and the number of concurrent jobs. The other controllers use a heuristic algorithm to adjust the number of resource units on the basis of both CPU and disk IO usage by jobs. To manage the maximum number of available resource units in each node, the controllers also take resource usage by other processes (e.g., system processes) into account. A prototype of our approach was implemented for Apache Hadoop on a cluster running at CloudLab. The proposed approach was demonstrated and evaluated with workloads composed of jobs with similar resource usage patterns as well as other realistic mixed-pattern workloads synthesized by SWIM, a statistical workload injector for MapReduce. The evaluation shows that the proposed approach yields up to a $48 \%$ reduction of the jobs makespan that results from using Hadoop-default settings.
\end{abstract}

CCS Concepts: • Theory of computation $\rightarrow$ Self-organization; • Computing methodologies $\rightarrow$ Distributed computing methodologies; • Computer systems organization $\rightarrow$ Self-organizing autonomic computing;

Additional Key Words and Phrases: Performance tuning, self-tuning, fuzzy control, makespan, data-analytics frameworks, autonomic control, job completion time, resource utilization, Hadoop, MapReduce, Capacity Scheduler

This work is supported in part by the National Science Foundation (NSF) grant No. 1234983 and the AT\&T Foundation. Authors' addresses: G. J. Lee and J. A. B. Fortes, Electrical and Computer Engineering Department, University of Florida, P.O. Box 116200, 339 Larsen Hall, Gainesville, FL 32611-6200; emails: \{giljael, fortes\}@acis.ufl.edu.

Permission to make digital or hard copies of all or part of this work for personal or classroom use is granted without fee provided that copies are not made or distributed for profit or commercial advantage and that copies bear this notice and the full citation on the first page. Copyrights for components of this work owned by others than ACM must be honored. Abstracting with credit is permitted. To copy otherwise, or republish, to post on servers or to redistribute to lists, requires prior specific permission and/or a fee. Request permissions from permissions@acm.org.

(c) 2019 Association for Computing Machinery.

1556-4665/2019/03-ART13 \$15.00

https://doi.org/10.1145/3309539 


\section{ACM Reference format:}

Gil Jae Lee and José A. B. Fortes. 2019. Improving Data-Analytics Performance Via Autonomic Control of Concurrency and Resource Units. ACM Trans. Auton. Adapt. Syst. 13, 3, Article 13 (March 2019), 25 pages. https://doi.org/10.1145/3309539

\section{INTRODUCTION}

Data-analytics services increasingly need to support multiple concurrent jobs and users. Several frameworks have been developed to enable scalable multi-tenant implementations of these services, a most noteworthy example being the Hadoop framework [24]. A general characteristic of these frameworks is a resource management approach that relies on pre-defined resource units to partition resources among multiple jobs. A resource unit is an abstract notion to represent a collection of physical resources, e.g., a unit could correspond to 1 CPU core and 1GB RAM (different versions of Hadoop use the term slot or container to refer to resource units). Usually the size of a unit and the total number of units available in the cluster are static in nature, i.e., determined prior to cluster creation and/or execution of jobs and unchanged during cluster operation and while jobs are running.

This form of static resource management has a limited ability to cope with increasingly diverse jobs and dynamic computing environments, resulting in low and hard-to-predict performance and poor resource utilization. This article proposes programmatic approaches to change configuration parameters dynamically in order to improve resource usage and job performance.

Data-analytics frameworks provide configuration parameters that are tunable to improve performance significantly [7]. Two examples of parameters are the maximum available number of concurrent jobs and the maximum available number of resource units. Users and system administrators can manually tune jobs or frameworks, following some recommendations, sometimes referred to as "the rules of thumb" [8]. However, these recommendations are for limited jobs and circumstances. Manual tuning of parameters is difficult and time-consuming because frameworks have many mutable parameters which also have complex inter-dependencies. For example, Hadoop has over 200 tunable parameters [26]. In addition, manual tuning may require knowledge that is specific to jobs and/or systems. To address limitations of manual tuning, self-tuning methods have been proposed.

Most self-tuning approaches optimize configuration parameters for a given job or framework. Such optimizations rely on first constructing performance models and then looking for modelbased near-optimal configurations $[6,7,9,11,14,22,27,28]$. However, those methods require time-consuming model-training phases. When the targeted jobs, their input datasets, or analytics frameworks are changed, it is necessary to reconstruct or retrain the models. For example, new Hadoop versions have been released at least twice every year, which would require such models to be reconstructed frequently. On the other hand, there are some proposals that focus on controlling resource usage by jobs to improve performance $[10,12,30]$. They seek to improve overall performance by avoiding over-/under-allocation of resources to jobs. Approaches based on resource usage can provide optimizations that are relatively job-independent and less affected by dynamics of jobs and execution environments. However, they have a limited ability to cope with dynamics of jobs when failing to also consider usage in terms of the number of resource units and its dynamic management.

This article introduces a Hierarchical Self-tuning Architecture (HSA) to improve upon unitbased static resource management in data-analytics frameworks and to overcome the following limitations of existing self-tuning approaches for performance improvement: (1) need for a time-consuming model-training phase, (2) unsuitability for environments that run multiple 
concurrent jobs, and (3) need for modification of either the framework or jobs or both. The HSA combines hierarchically the control of cluster-wide job concurrency and the control of per-node maximum number of units (hereon referred to as MaxNumUnits) in order to improve performance without modification of either the framework or the jobs. The choice of these parameters is based on experimental evidence and empirical studies from past works [10, 12, 30]. The architecture comprises four main types of components: the global controller (of job concurrency), the global monitor (of aggregate resource usage), the per-node local controllers (of MaxNumUnits), and the local monitors (of resource usage per node). For the global controller, a fuzzy-logic [29] -based controller is proposed. Fuzzy rules are generated using a concave-downward relationship between aggregate resource usage and the maximum number of concurrent jobs (hereon referred to as MaxNumJobs). Each of the local controllers uses a similar heuristic algorithm, which seeks full resource utilization in a local node. Each local monitor collects local resource-usage information that includes resource usage by jobs as well as other processes (e.g., system processes). Based on the resource-usage information, the local controller dynamically adjusts the per-node MaxNumUnits. The global monitor aggregates physical-resource (e.g., CPU and IO) usage as well as unit-usage information from the local monitors. The global controller uses the aggregate resource-usage information to dynamically control MaxNumJobs [3]. A prototype of the HSA was implemented and applied to the Apache Hadoop framework, a widely used open-source implementation of MapReduce (MR). The local controllers of the prototype consider both CPU and disk IO usage in order to manage MaxNumUnits. The prototype implementation on a cluster running at CloudLab [17] demonstrates that HSA is applicable for workloads composed of jobs that have resource-usage characteristics similar to those of the Hadoop examples used in the experiments as well as for realistic workloads synthesized by a statistical workload injector for MapReduce (SWIM) [1]. HSA achieves an average improvement of $40 \%$ in CPU utilization and up to $48 \%$ reduction in jobs' makespan when compared to running the workloads with Hadoop-default settings. The key contributions and innovative aspects of the approach in this article are as follows:

-Experimental evidence of the limitations of static unit management in data-analytics frameworks. Different settings of MaxNumUnits lead to different performance.

- Characterization of the relationship between MaxNumJobs and performance.

- Experimental observation of a concave-downward relation between MaxNumJobs and CPU usage.

-A job-concurrency controller using fuzzy-logic management rules based on the concavedownward relation between MaxNumJobs and CPU usage.

- A heuristic algorithm based on resource usage; it is used by local controllers to adjust pernode MaxNumUnits according to resource usage (e.g., CPU and disk IO in the prototype implementation).

- A prototype of the HSA implemented on Hadoop 3.0.0-alpha2 (Hadoop 3); it shows that the HSA yields significant performance improvement over Hadoop-default configurations.

The remainder of this article is organized as follows: Section 2 provides background and motivation for this article's research. Section 3 studies the correlation between resource usage and the maximum number of units and concurrent jobs, and demonstrates how the number of concurrent jobs affects the overall performance of a Hadoop cluster. Section 4 introduces the HSA. Section 5 describes the prototype implementation of the HSA on the Hadoop 3 framework. Section 6 presents the experimental evaluation of the HSA with Hadoop representative workloads and realistic workloads. Section 7 discusses related work, and Section 8 concludes the article. 


\section{BACKGROUND AND MOTIVATION}

\subsection{Fuzzy Control}

Fuzzy control (also known as fuzzy-logic control) is based on fuzzy logic. A fundamental concept is that of a fuzzy set where each element has a membership value given by a membership function in a continuous interval between 0 and 1 . Fuzzy control is composed of four elements: a fuzzy rule-base, a fuzzy inference mechanism, a fuzzification interface, and a defuzzification interface. The fuzzy rules in the rule-base are represented as "If antecedents Then consequents" where antecedents are expressions involving fuzzy sets. Fuzzy rules quantify a linguistic description of how the target system is controlled. The fuzzification process converts controller inputs, usually numerical values, into fuzzy values. The fuzzy inference mechanism uses fuzzy rules to map these fuzzy values into outputs that are also fuzzy values. These outputs are converted into numerical values that can be applied to the target system (this process is called defuzzification). The underlying assumption in the proposed approach is that various jobs are submitted to the cluster, so it is difficult to have prior knowledge about the aggregate jobs (e.g., resource-usage patterns) before executing them. Hence, this article suggests generic fuzzy rules based on the correlation between resource usage and concurrent jobs-the global controller uses these fuzzy rules to dynamically adjust MaxNumJobs. Extensive discussion of fuzzy control can be found in [29].

\subsection{YARN}

Resource management, job scheduling, and monitoring in YARN use three types of daemons: a global ResourceManager (RM), a per-job ApplicationMaster (AM), and a per-node slave NodeManager (NM). The ResourceManager includes Scheduler and ApplicationsManager as components. The Scheduler allocates resource units (each corresponding to a so-called container in YARN), according to requirements of each job. The Scheduler operation is determined by a pluggable scheduler. The current MR plug-in schedulers are the Capacity Scheduler and the Fair Scheduler, the default being the Capacity Scheduler. The ApplicationsManager accepts job submissions, negotiates units for executing AMs for jobs submitted, and restarts the AMs to recover in case of failure. Once an AM for a job is launched, it is responsible for managing the tasks of the job. Each Hadoop job is composed of a single AM and a set of tasks. The NM is responsible for units, monitoring, and reporting units' resource usage to the RM. Figure 1 summarizes the main steps needed to execute a job on YARN.

The Capacity Scheduler [18] is designed to enable multiple tenants to share a large cluster with per-tenant capacity guarantees. The scheduler provides resource elasticity so that free resources can be allocated to any queues beyond their capacity constraints, but when the original queue that owns the free resources demands them, the free resources allocated to other queues are re-allocated to the original queue. It has a dynamically adjustable configuration parameter-yarn.scheduler.capacity.maximum-am-resource-percent, AMRP-which limits the maximum amount of resources being used by AMs. Hence, this parameter can be used to control the number of concurrent active jobs. For example, AMRP $=0.6$ means that up to $60 \%$ of resources in the cluster can be used to run AMs. The default value is AMRP $=0.1$. In the case when primarily small-sized jobs (e.g., a job consisting of one mapper and one reducer) run in the cluster, high AMRP may improve performance. On the other hand, when there are mainly large-sized jobs, high AMRP may increase response time because, among other reasons, several of the AMs already launched may still be waiting for units to become available and be allocated to their tasks and preventing AMs of running jobs from having additional units allocated to their tasks (i.e., unit allocation to jobs being activated may result in unit starvation for other already active jobs).

This article considers the case when the default Capacity Scheduler is used in the reference Hadoop system (i.e., the system against which the proposed approach is evaluated and on 


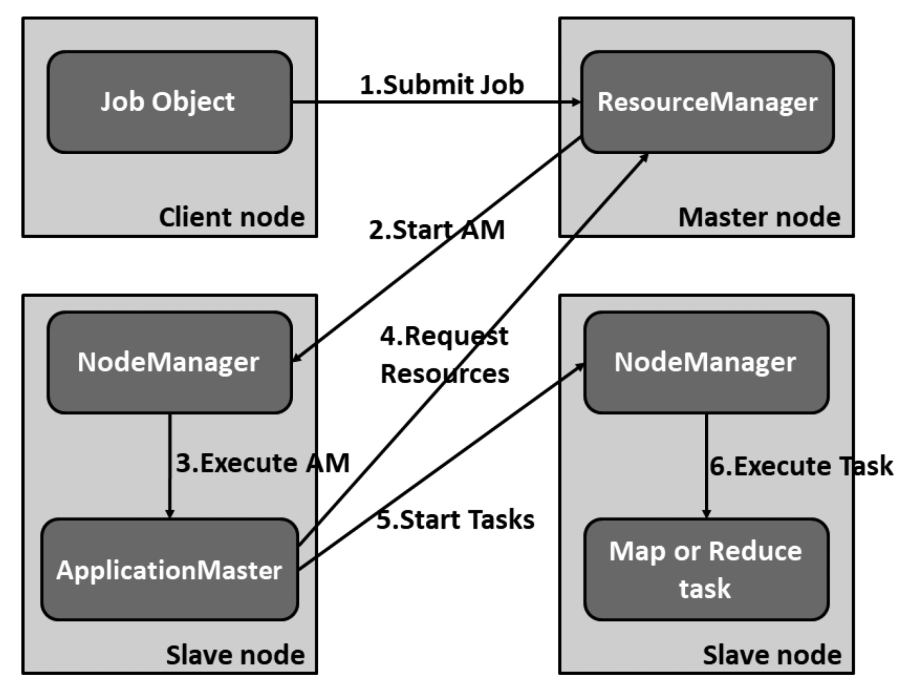

Fig. 1. Simplified sequence of steps to launch a job, starting from job submission and ending in execution of the tasks of a job on YARN resource units. (1) A client submits a job by interacting with a job object which runs inside a JVM. Prior to the job submission to the RM, job metadata such as the job ID is acquired from the RM and all the job-related resources such as jar files and configurations are moved to the distributed file system (e.g., HDFS) to make them globally available for the rest of the job execution. (2) the RM chooses a NM and requests units for the job's AM. (3) The NM allocates units for the AM and launches it. (4) The AM requests from the RM the resources needed for execution of the tasks of the job. (5) The AM tells the NMs allocated by the RM to start their assigned tasks. (6) NMs launch tasks of the job.

which the approach is implemented) and characterizes the relation between resource usage and MaxNumJobs. In this context, MaxNumJobs is represented by AMRP in accordance with the equation, MaxNumJobs $=$ MaxNumUnits $\times$ number of slave nodes $\times$ AMRP/Units per AM. Based on this understanding, a self-tuning method that updates the value of MaxNumJobs dynamically (rather than using a static value) is proposed. Additional information on YARN can be found in [24].

\subsection{Static Management}

Static settings of parameters that determine job concurrency and (the number of) resource units are unlikely to deal effectively with the diversity of analytics jobs, the dynamics of a job's resource demands, the variety of possible configurations, and the heterogeneity of resources. To alleviate these limitations, some of the analytics frameworks support dynamic features so that users or administrators can update MaxNumJobs on the fly. For example, in the Hadoop system, MaxNumJobs can be controlled by changing the value of AMRP which indirectly captures MaxNumJobs as a percentage of units usable for AMs. Nevertheless, most such management operations entail ad-hoc manual human intervention rather than programmatic and continuous actions, thus being neither scalable nor efficient.

In addition, for effective management of resources (e.g., CPU and memory), analytics frameworks commonly define a unit of resources, sometimes called slot or container, which is an abstract notion to represent a collection of physical resources (e.g., a unit could correspond to $1 \mathrm{CPU}$ core and 1GB RAM). The number of units assigned to a job is determined from the job's resource demand. The units can be preassigned exclusively to a specific type of tasks (e.g., map or reduce in Hadoop) or to any types of tasks. Intuitively, the task-specific unit assignment can cause lower resource utilization than the general-type unit assignment when there are tasks waiting for units 
Table 1. Jobs Used for Experiments

\begin{tabular}{cccc}
\hline Job & $\begin{array}{c}\text { Resource-usage } \\
\text { pattern }\end{array}$ & $\begin{array}{c}\text { Number of tasks } \\
\text { (Map: Reduce) }\end{array}$ & Generator of Dataset (Size) \\
\hline Terasort (TS) & IO-intensive & $17(16: 1)$ & Teragen (2GB) \\
Grep (GR) & CPU-intensive & $39(37: 2)$ & Randomtextwriter (4GB) \\
Wordcount (WC) & Balanced CPU and IO & $28(27: 1)$ & Randomtextwriter (3GB) \\
\hline
\end{tabular}

The resource-usage types are classified on the basis of CPU utilization and Write-IO activity [6].

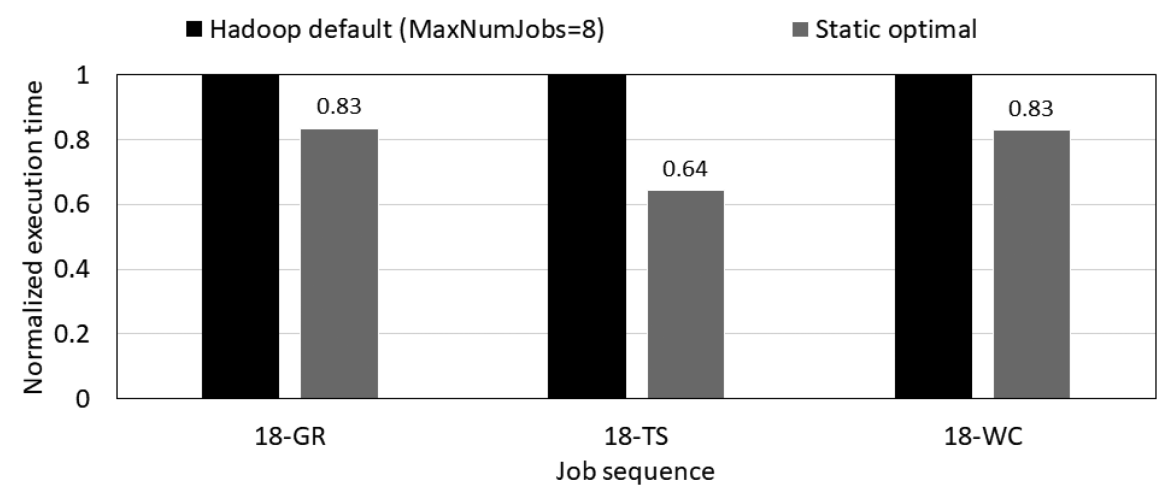

Fig. 2. Normalized execution times of job sequences 18-TS, 18-GR, and 18-WC using the Hadoop-default value of MaxNumJobs ( 8 in the 10-slave cluster) and the optimal static value of MaxNumJobs (24, 40, and 16, respectively) for each job sequence.

of a specific type to become available while units of different types are available to be assigned. In addition, the maximum number of units per node (i.e., MaxNumUnits in the context of Hadoop) is determined statically by cluster administrators, generally in the initialization phase (e.g., following rules of thumb in the case of YARN [8]).

\section{STATIC CONTROL AND PERFORMANCE}

To study the relationship between MaxNumJobs, MaxNumUnits, and performance, three examples of Hadoop jobs (hereon simply called jobs) are selected according to their resource-usage characteristics: Terasort (IO-intensive), Grep (CPU-intensive), and Wordcount (balanced CPU-IO). Three different sequences of Terasort, Grep, and Wordcount jobs are generated: 18-TS (18 Terasort jobs), 18-WC (18 Wordcount jobs), and 18-GR (18 Grep jobs). Table 1 shows information of each job selected and its input dataset. The value of MaxNumJobs is set statically. The experiments reported in this section were conducted on a cluster (1 master and 10 slaves) composed of 11 physical nodes on the m510 CloudLab cluster (Section 6.1 describes the hardware specifications of each node). Apache $^{\mathrm{TM}}$ Hadoop ${ }^{\circledR}$ release 3.0.0-alpha2 (25 January 2017) is installed on the cluster. The master node runs the ResourceManager for YARN and NameNode for the Hadoop Distributed File System (HDFS). Each slave node runs the NM for YARN and the DataNode for HDFS. The HDFS replication factor is 3 and block size is $64 \mathrm{MB}$. All experiments use Hadoop-default configurations.

Figure 2 shows the makespan of each job sequence for both the Hadoop default and optimal MaxNumJobs. The optimal MaxNumJobs was found via exhaustive search and experimentation. Changing MaxNumJobs to its optimal value improved performance significantly-up to 36\% (in the case of 18-TS). Each job sequence has a different optimal MaxNumJobs. 


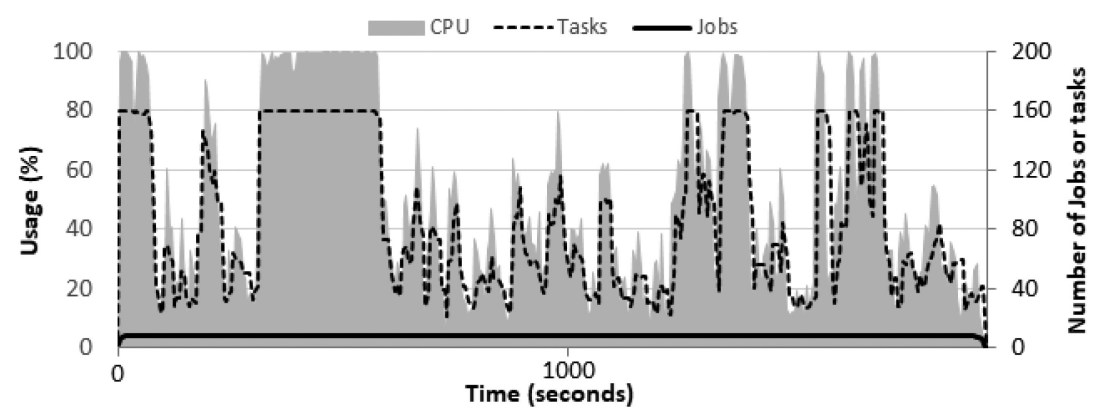

(a)

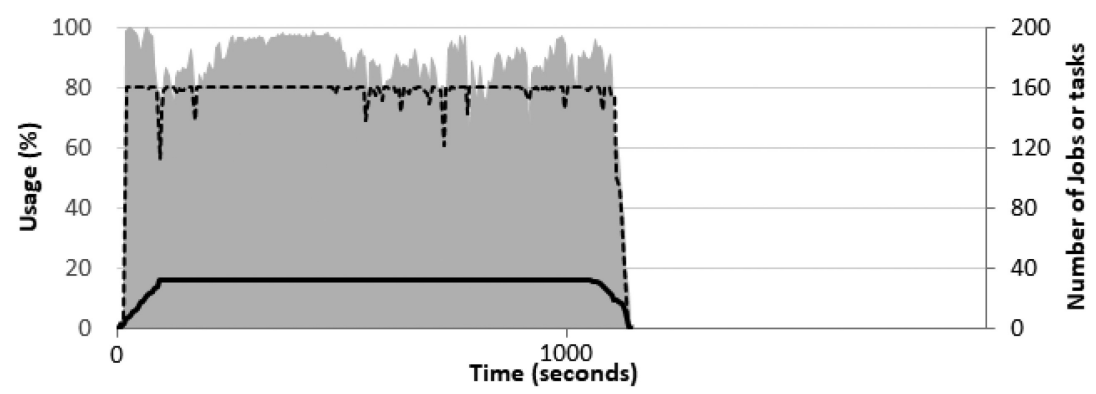

(b)

Fig. 3. CPU usage (CPU), the number of running jobs (Jobs), and the total number of active units (Tasks) while the 200-SWIM sequence runs in Hadoop. (a) Makespan = 1,876 seconds for MaxNumJobs $=8$ (default) and MaxNumUnits = 16. (b) Makespan = 1,129 seconds for MaxNumJobs = 32 and MaxNumUnits = 16 . Arrows in (a) convey a change of MaxNumJobs from 8 (the solid line) to 32 (the thick dashed line), which results in the improved CPU utilization shown in (b).

MaxNumJobs also affects performance when the job sequence has mixed types of jobs. Figure 3 shows that MaxNumJobs choices can improve performance (i.e., makespan) significantly (40\%). This experiment was conducted with a 200-job sequence. The jobs are selected from the biggest jobs among workloads generated by SWIM. Hadoop can use $10 \%$ of units for AMs by default. Hence, in the 10 -slave cluster which consists of 160 units, $8\left(=160_{\text {units }} \times 0.1_{\mathrm{AMRP}} / 2_{\mathrm{Unit}}\right.$ per AM $)$ is the default MaxNumJobs that the Capacity Scheduler can launch simultaneously. Likewise, when $40 \%$ of units can be used for AMs, MaxNumJobs is $32\left(=160_{\text {units }} \times 0.4_{\mathrm{AMRP}} / 2_{\text {Unit per AM }}\right)$. As shown in Figure 3(a), though there are available units during the entire running time and many jobs are waiting to be scheduled in the queue (not shown in the figure), launching additional jobs is constrained by MaxNumJobs, resulting in low CPU utilization. Hence, as shown in Figure 3, increasing MaxNumJobs can improve CPU utilization.

To illustrate the limitations of static-based resource management, we measured Hadoop's performance on the 200-job sequence for different values of two configuration parameters: MaxNumJobs and MaxNumUnits. All the parameters are set statically and kept unchanged during each experiment.

Figure 4(a) shows how the makespan of the 200-job sequence decreases as MaxNumJobs increases. Moreover, different settings of MaxNumUnits result in different performance. For example, when MaxNumJobs is set to use $50 \%$ of units in the 10-slave cluster and MaxNumUnits is set to 20, a total of 200 units are available in the Hadoop cluster and up to 100 out of 200 units can be used for AMs. That is, at most 50 AMs can run at the same time (by default, YARN assigns two units to each AM). Though the 200 jobs in the sequence are chosen from the biggest jobs generated by SWIM, 

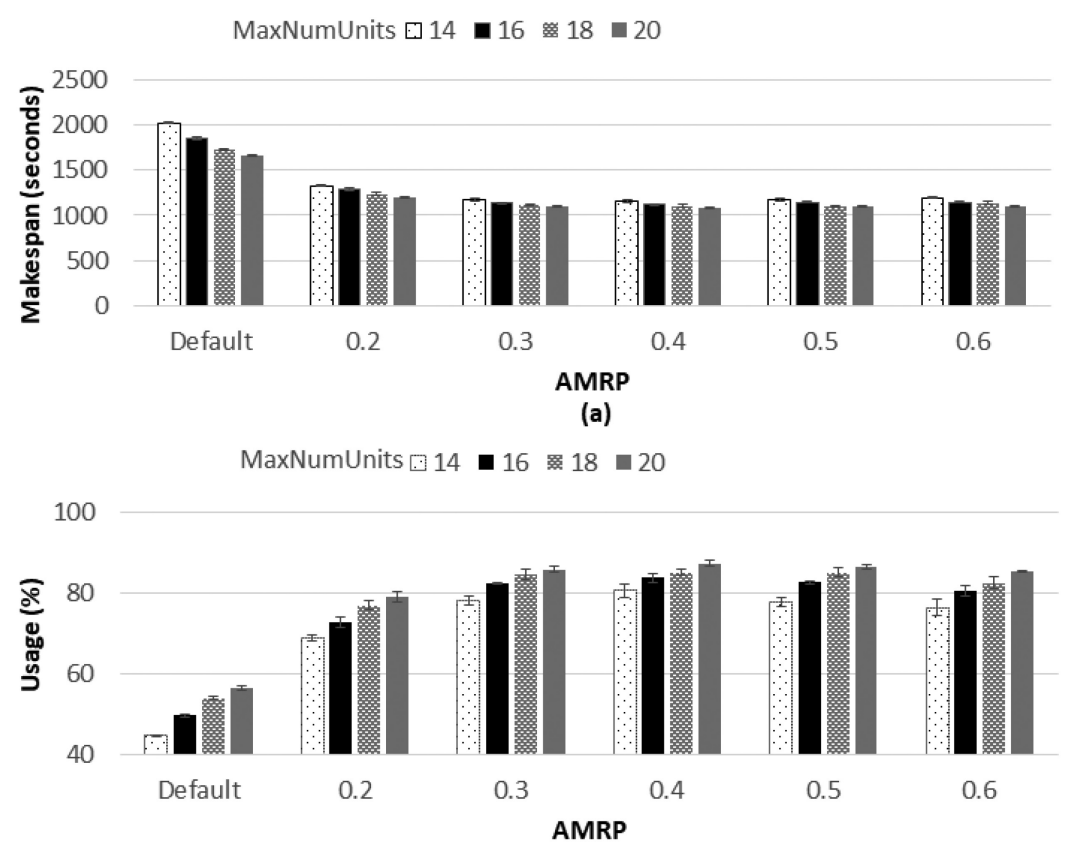

(b)

Fig. 4. (a) Makespan of a 200-SWIM sequence for different MaxNumUnits (14, 16, 18, and 20) and AMRP, where AMRP indirectly represents MaxNumJobs $=$ number of slave nodes $\times$ MaxNumUnits $\times A M R P / 2$. (b) CPU usage for different MaxNumUnits and MaxNumJobs.

most of them are too small to occupy many units in the Hadoop cluster (scheduling consecutive small jobs has overhead and [16] addresses this issue). Therefore, increasing both MaxNumJobs and MaxNumUnits can decrease the makespan of the job sequence. This result shows that optimal performance is hard to achieve by simply following rules of thumb for static configuration of Hadoop parameters.

Figure 5(a) shows how, when MaxNumJobs = 32 and MaxNumUnits = 16, all available units in the Hadoop cluster are likely to be utilized. Nevertheless, CPU resources are not fully utilized. As demonstrated in [12], the number of concurrent tasks is highly correlated with CPU usage. Hence, it is expected that an appropriate increase of the number of tasks can improve CPU utilization, further increasing overall performance. Figure 5(b) illustrates how an increase of MaxNumUnits $(=20)$ helps improve CPU utilization, though the resulting makespan reduction is not big $(2 \%)$ when compared with Figure 5(a) in the environment used for this experiment.

On the other hand, Figure 6 shows that simply increasing or decreasing MaxNumUnits does not guarantee better performance. The figure shows the completion time of the biggest of the 200 jobs when it runs alone in the 10-slave Hadoop cluster with different initial values of MaxNumUnits. As MaxNumUnits decreases, job completion time also decreases up to 12 units per node. However, when MaxNumUnits is set to 8, it shows the worst performance. This job is CPU-intensive during the map phase and becomes IO-intensive when mappers locally store intermediate results before the shuffle phase. This shows that achieving optimal performance even for a single job may require deep understanding of the job itself and time-consuming experiments to search for good parameter configurations.

In summary, when MaxNumJobs and MaxNumUnits are set statically to the best of the experimentally determined values (i.e., MaxNumJobs $=40$ and MaxNumUnits $=20$ ), a reduction of $42 \%$ 

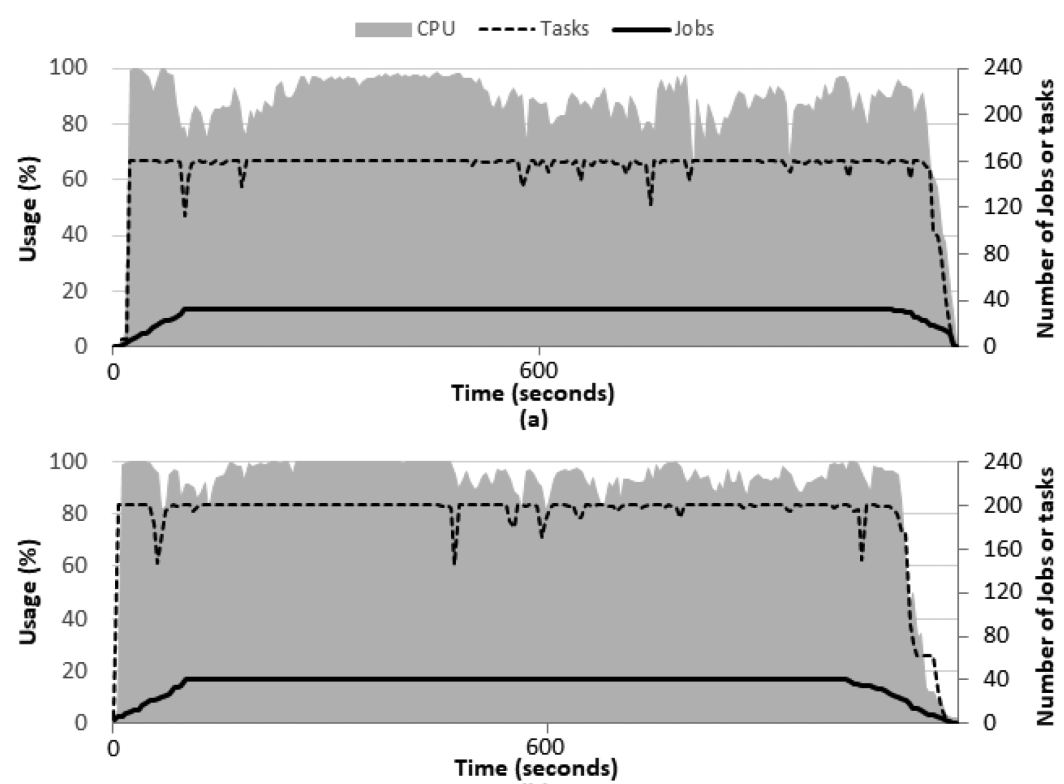

(b)

Fig. 5. CPU usage versus the number of running jobs (Jobs) and versus the total number of units in use (Tasks) while running the 200-SWIM sequence. (a) Makespan = 1,129 seconds for MaxNumJobs $=32$ and MaxNumUnits $=16$. (b) Makespan $=1,093$ seconds for MaxNumJobs $=40$ and MaxNumUnits $=20$. Arrows in (a) convey an increase in concurrency by changing MaxNumUnits from 16 to 20 (the thick dashed line) in order to increase CPU usage and (b) shows the result.

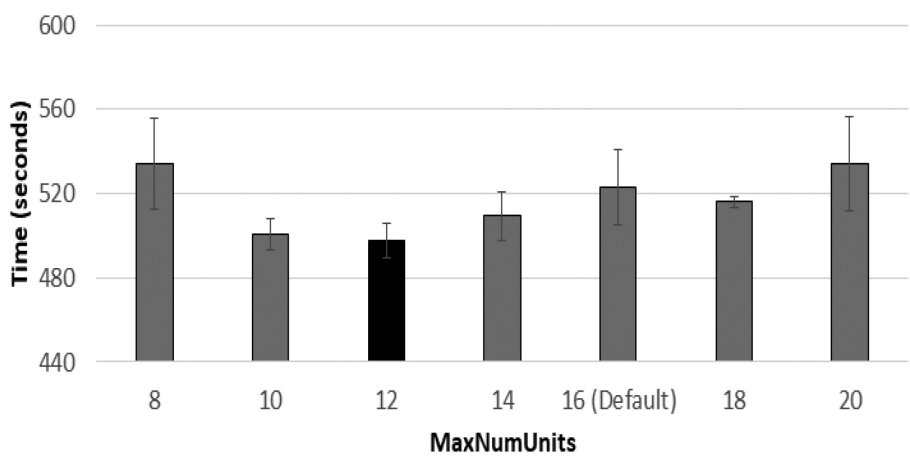

Fig. 6. Job completion time of a single job for different values of MaxNumUnits. MaxNumUnits $=16$ is the value recommended by rules of thumb for the system used for this experiment.

of the makespan is achieved, compared with the default case of MaxNumJobs $=8$ and MaxNumUnits $=16$ (as shown in Figures 3 and 5). However, it is impractical to determine experimentally the optimal static settings for each possible set of jobs and execution dynamics. These observations provide intuition and partial justification for the desirability of the dynamic adjustment of MaxNumJobs and MaxNumUnits to effectively accommodate different types and mixes of jobs as well as runtime dynamics. This article provides online methods that dynamically adjust MaxNumJobs and MaxNumUnits on the basis of observed resource usage, in an autonomic way. 


\section{HIERARCHICAL SELF-TUNING APPROACH}

HSA adapts the configuration of the analytics framework to the diversity of jobs and the dynamics of their resource demands. With respect to the variety of possible configurations, MaxNumJobs and MaxNumUnits are the only parameters whose values are adjusted as jobs are being executed. For simplicity, we assume that the cluster is composed of identical slave nodes and leave the heterogeneous case (i.e., different slave nodes) to future research. We believe that the proposed approach generalizes to the heterogeneous case by building on the idea of modeling faster or higher-capacity resources as multiple slower or smaller resources of a given type, respectively, thus converting the heterogeneous case to the homogeneous one. However, further research needs to be done to work out details and validate this claim, including the use of schedulers that are best suited for heterogeneous Hadoop [31]. HSA assumes that various jobs (whose workloads are unknown) are submitted and share the resources of a cluster while being executed. It seeks to achieve high cluster resource utilization (and consequently to reduce makespan) without modifying either the job codes or the analytics frameworks. Hence, this architecture is applicable to any analytics framework that satisfies two requirements: (1) it is possible to monitor resource usage in slave nodes and (2) it is possible to control the total number of jobs and the number of resource units per node.

In this article, the performance objective is the makespan of a sequence of jobs to be executed. The proposed approach seeks to minimize makespan by adjusting MaxNumJobs and MaxNumUnits at any given time so as to increase resource usage. However, the makespan of a job sequence is not observable until the job sequence finishes and therefore is not useful for feedback-based control during job-sequence execution. Alternatively, resource usage is observable and useful as a feedback measure since it is highly correlated with makespan [12]. Intuitively, the higher the resource usage the faster the jobs progress, resulting in faster overall job execution. Therefore, minimizing makespan (Figure 4(a)) can be considered as maximizing resource usage (Figure 4(b)) at time $t$ by adjusting MaxNumJobs and MaxNumUnits.

As shown in Figure 7, the HSA has four components: global controller, global monitor, local controller, and local monitor. The resource manager and node managers are typical components of analytics frameworks. The resource manager is also called scheduler because it schedules jobs and assigns resources to the jobs. As previously mentioned, each job is made up of one or more tasks. Node managers manage local resources and launch tasks according to the resource manager's requests. The global controller controls job concurrency while the local controllers manage task concurrency by controlling the number of resource units available to run tasks. The roles of the HSA components and processes are described below in detail.

Global Monitor: The global monitor runs in the master node. It aggregates resource-usage information from each local monitor (explained below) and calculates overall resource usage. The value of aggregate resource usage is an input to the global controller. The global monitor collects unit-usage information by all slave nodes directly from the scheduler and collects the same information from the local monitors in the slave nodes. This information includes how many units are in use and how many are free. The two values of aggregate unit usage (one obtained from the scheduler and the other from the local monitors) are not necessarily identical because the global monitor communicates with the local monitors asynchronously. The global monitor checks if the two values match and communicates the result of the check to the local monitors for future use.

Global Controller: The global controller plays a critical role in controlling MaxNumJobs in the cluster. It assumes a concave-downward relationship between MaxNumJobs and performance, which is deduced from the observation of how adjusting MaxNumJobs decreases makespan (as shown in Figure 4(a)). Each task of a job is a part of the job's overall workload, thus the increase or decrease of MaxNumJobs is correlated with the increase or decrease of the number of concurrent tasks. Intuitively, too small a number of jobs and too large a number of jobs can lead to poor 


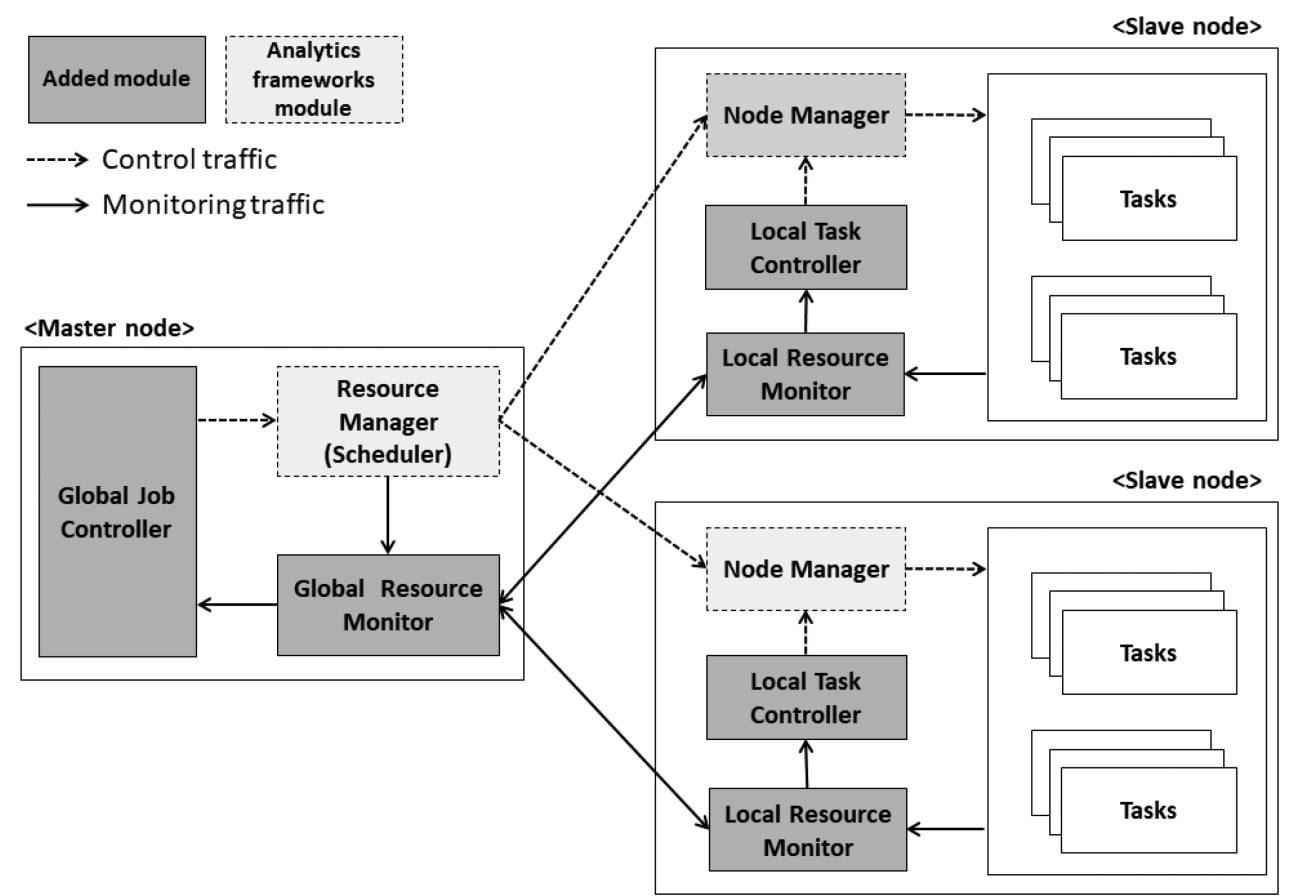

Fig. 7. Hierarchical self-tuning architecture (HSA) for performance improvement by controlling the numbers of jobs and units in data-analytics frameworks.

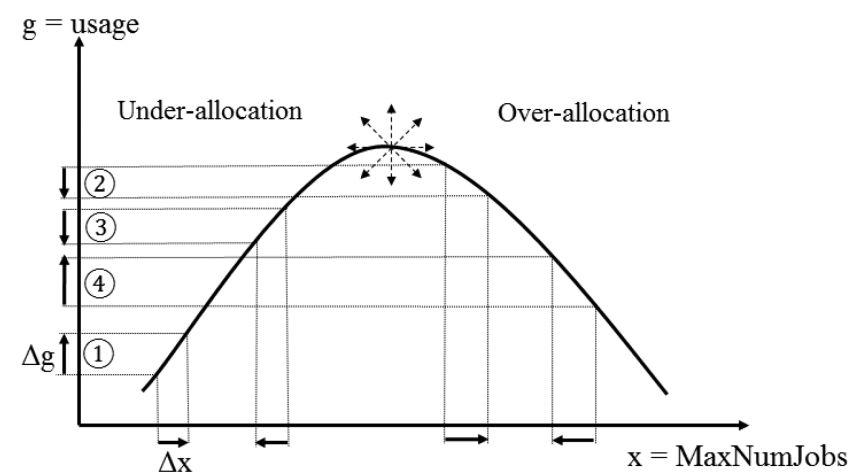

Fig. 8. Resource usage (e.g., CPU) as a concave-downward objective function of $x=$ MaxNumJobs. The cluster may run various jobs simultaneously, so the operating point may change over time in one of the directions illustrated by the dotted arrows. Circled numbers 1-4 correspond to the antecedents of rules in Table 2.

resource usage due to under-utilized idle units waiting on task assignments or overutilized units due to the use of resources by too many AMs, respectively. The concave-downward relationship of Figure 8 illustrates this intuition. Examples of real data samples depicting this relationship are shown in Figure 4(b).

As illustrated in Figure 8, when too few (under-allocation) or too many (over-allocation) jobs run concurrently, practical resource utilization decreases, resulting in performance degradation. For example, in Hadoop with a static value of MaxNumUnits, running more jobs may not always 


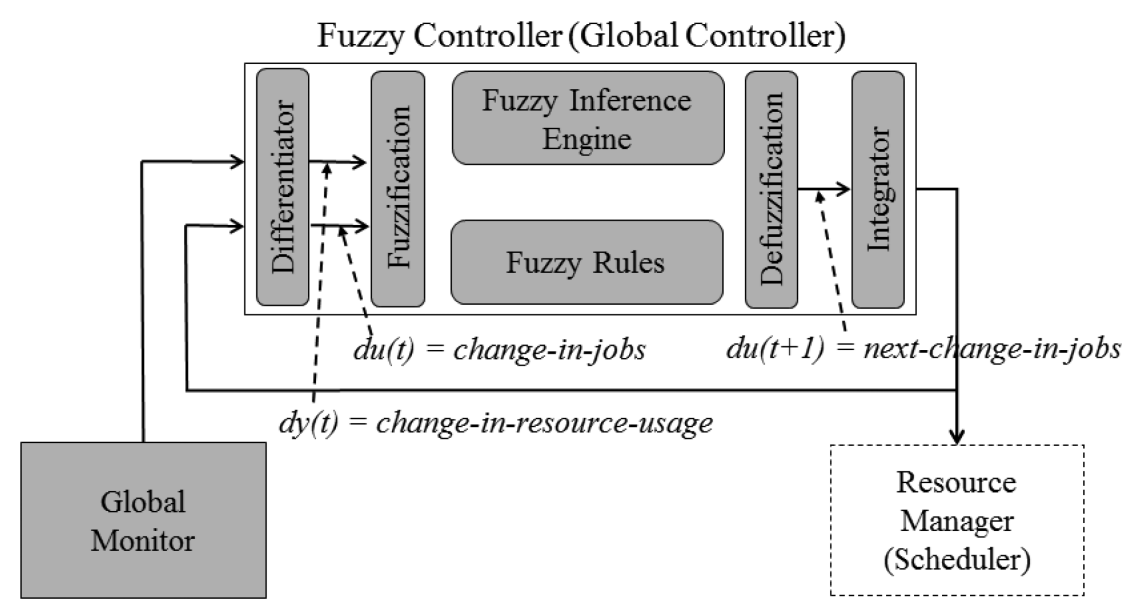

Fig. 9. Fuzzy-logic-based global controller. It uses inputs provided by the global monitor and provides outputs to the scheduler.

increase resource utilization. Hadoop may assign units to some AMs but be unable to assign units to the tasks managed by the AMs. These AMs use units but do little or no processing while waiting for additional units to be available for their tasks, lowering resource utilization. The presence of many such AMs when over-allocating units can thus cause lower resource utilization. Furthermore, as it is assumed that arbitrary jobs are submitted, and the jobs' resource demands may be dynamic, the nature of submitted jobs is likely to contribute to changes of the optimal value of MaxNumJobs. Therefore, continuous performance optimization is required on the basis of monitored resource usage.

As already discussed, the dynamics of jobs make it hard to create a generic performance model. To address this problem, we propose a job-concurrency controller based on fuzzy logic. It comprises fuzzification and defuzzification interfaces, fuzzy rules, fuzzy inference engine, differentiator, and integrator, as shown in Figure 9. It employs the following linguistic variables: change-in-jobs, change-in-resource-usage, and next-change-in-jobs. Change-in-jobs is the change of MaxNumJobs in successive sample times. Change-in-resource-usage is the difference of actual resource usage at a given sample time and at the previous sample time. Next-change-in-jobs is the change in the percentage of units available to launch jobs at from the current sample time to the next sample time. The fuzzy controller requests the resource manager to adjust MaxNumJobs, delivering next-change-in-jobs. The fuzzy controller receives two inputs at time $t$ from its output and cluster-wide resource usage calculated in the global monitor. The differentiator computes the differenced control input (change-in-jobs) $d u(t)=u(t)-u(t-1)$ and the differenced output (changein-resource-usage) $d y(t)=y(t)-y(t-1) . d u(t)$ and $d y(t)$ are inputs to the fuzzification interface and they are translated to linguistic values.

The actions of the fuzzy controller follow the fuzzy rules in its rule-base which are based on the concave-downward relationship between MaxNumJobs and resource usage (see Section 3). The fuzzy controller updates MaxNumJobs via control actions such as increasing and decreasing the value of a concurrency-control parameter (AMRP in Hadoop). For the linguistic values, "negative," "positive," and "zero" are used and they can be abbreviated to NE, PO, and ZE, respectively. Figure 10 shows the membership functions for the input and output variables. For simplicity's sake, a triangular-shaped curve for ZE and a trapezoidal-shaped curve for NE and PO are used as membership functions. For fine-grained control, the membership functions could be made more elaborate 


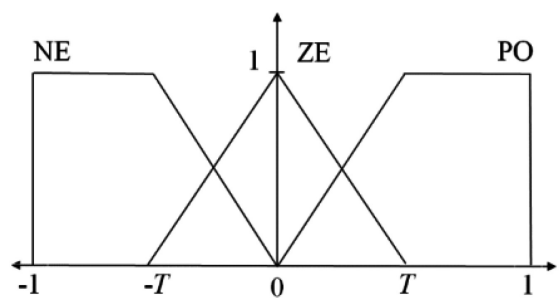

Fig. 10. Membership functions for input and output. $T$ and $-T$ are the $x$-intercepts of the triangular membership function. $T=0.5$ in our experiments.

Table 2. Main Fuzzy Rules of the Fuzzy Controller Derived from Figure 8

\begin{tabular}{|c|c|c|c|}
\hline \multirow{2}{*}{ Rule } & \multicolumn{2}{|c|}{ IF } & THEN \\
\cline { 2 - 4 } & $\begin{array}{c}\text { change-in-jobs AND } \\
\text { change-in-resource-usage }\end{array}$ & next-change-in-jobs \\
\hline $\mathbf{1}$ & $P O$ & $P O$ & $P O$ \\
\hline $\mathbf{2}$ & $P O$ & $N E$ & $N E$ \\
\hline $\mathbf{3}$ & $N E$ & $N E$ & $P O$ \\
\hline $\mathbf{4}$ & $N E$ & $P O$ & $N E$ \\
\hline
\end{tabular}

by adding other linguistic values and fuzzy rules. However, this may make the controller more sensitive to small changes, so the chosen fuzzy controller uses only the three above-mentioned linguistic values.

Table 2 shows fuzzy rules generated for the proposed fuzzy controller, based on the fuzzy membership functions and the concave-downward relationship. Rules 1-4 are the main fuzzy rules of the fuzzy controller derived from (1) to (4) in Figure 8, respectively. Since various jobs can be submitted to the cluster, the operating point of the objective function may change over time. To deal with these changes more sophisticatedly, additional fuzzy rules can be added carefully. For example, IF change-in-jobs = ZE AND change-in-resource-usage $=P O$ THEN next-change-in-jobs $=Z E$ to avoid frequent updates of MaxNumJobs (this fuzzy rule is used in our experiments). The inference mechanism uses a $M I N(A, B)$ inference method (fuzzy AND operation) between inputs, and MAX $(A, B)$ inference method (fuzzy OR operation) between outputs of the rules [29]. The defuzzifier converts a linguistic value to a crisp value as output using the center-of-area method [23] which is widely used and generates the center of gravity of the possibility distribution of a control action. Finally, the crisp value is converted to a value that the analytics framework understands and can use to trigger a designated control action.

Local Monitor: The local monitor runs as a daemon in each slave node. It monitors overall usage of resources (e.g., CPU, memory, and IO) by all running tasks as well as all other processes (including system processes) in the slave node. It also collects unit-usage information. The two types of usage information are utilized by the local controller (explained below) to decide whether the current MaxNumUnits is appropriate or not. The monitored information is periodically sent to the global monitor so that the global monitor can calculate cluster-wide resource and unit usage.

Local Controller: The HSA seeks to control MaxNumUnits for performance improvement. This control is inspired by observations that full usage of all available units does not always result in full utilization of physical resources. Conversely, full usage of physical resources also does not always mean that all units are occupied. Generally, the granularity of units is determined by experts or rules of thumb (e.g., 1 CPU core and 1GB RAM per unit). To the best of our knowledge, there is 
no approach to configure or adjust dynamically MaxNumUnits in a system according to available resources per node while multiple jobs are running concurrently. The local controller continuously updates MaxNumUnits based on actually available resources in a slave node. To determine whether the current setting for MaxNumUnits is appropriate, it is necessary to evaluate whether resource contention exists. For this purpose, usage of resources such as CPU, disk IO, and network IO should be monitored, and their correlations need to be studied, but in this article only CPU usage and disk IO usage are considered and the others are left for future work.

In this article, each local (node) controller uses per-node CPU usage and disk IO usage information collected by the local monitor and decides to increase or to decrease MaxNumUnits in the slave node. For this purpose, the local controller uses a simple heuristic (Algorithm 1).

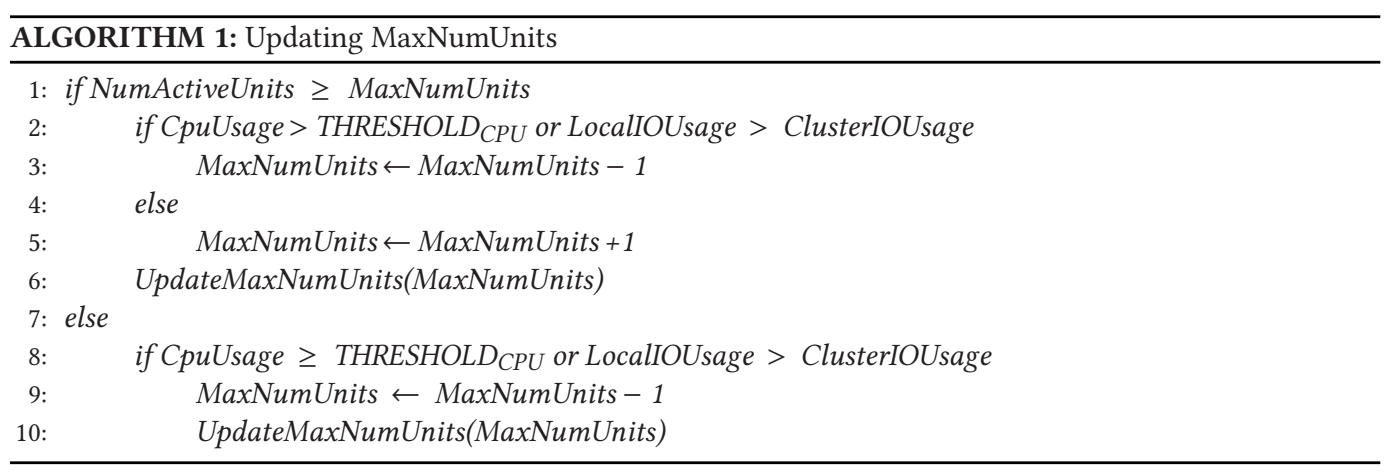

Generally, each node manager updates the scheduler on the node's unit-usage information periodically (e.g., every second in Hadoop). In the HSA, the global monitor collects the unit-usage information and then the local monitors fetch the aggregate information in the global monitor so that the local controllers can utilize it to make decisions. When aggregate unit-usage information collected from the scheduler matches the one collected by the local monitors, each local controller begins updating MaxNumUnits-this prevents too frequent updates of MaxNumUnits by the local controllers.

When there are no available units for tasks in a slave node (line 1), it is determined whether current MaxNumUnits is appropriate. If current CPU usage (CpuUsage) in the slave node is greater than the pre-determined threshold, THRESHOLD $D_{C P U}$, or current disk IO usage (LocalIOUsage) in the slave node is greater than average disk IO usage (ClusterIOUsage) in the cluster (line 2), MaxNumUnits is decreased to avoid resource over-utilization (line 3). It is hard to determine whether the measured amount of disk IO represents resource contention, so further research is necessary. For simplicity's sake, with respect to disk usage, the algorithm just compares LocalIOUsage with ClusterIOUsage to determine if MaxNumUnits is appropriate. ClusterIOUsage is the average disk IO usage of all the slave nodes in the cluster, calculated by the global monitor. MaxNumUnits is increased when there are available resources for additional tasks although all the units in the slave node are currently occupied by tasks (lines 4 and 5), which helps to increase resource utilization by giving more room for additional tasks.

On the other hand, MaxNumUnits can be updated when there are still available units in a slave node (lines 7-10). Full resource utilization can likely result from high contention among tasks. Hence, simply shrinking MaxNumUnits can help prevent such full resource utilization caused by tasks' contention when MaxNumUnits is set to a value greater than the capacity of the slave node. For example, when some tasks are CPU-intensive, CpuUsage can exceed its threshold value before the tasks take all units in a slave node. Hence, when CPuUsage is greater than THRESHOLD $D_{C P U}$ or 
LocalIOUsage is greater than ClusterIOUsage (line 8), this is the case when either MaxNumUnits is set to a value greater than the capacity of the slave node or some tasks are highly resource-intensive at a given time. The algorithm decreases MaxNumUnits to avoid such contention conditions (lines 9 and 10).

\section{IMPLEMENTATION}

We have implemented our prototype using Python and shell scripts on top of Apache Hadoop 3. In Hadoop 3, MaxNumUnits can be updated on the fly via APIs. This prototype implementation supports node-wide dynamic adjustment of MaxNumUnits as well as cluster-wide MaxNumJobs. The local monitor collects actual CPU usage and unit usage. All the collected information is delivered periodically to the global monitor so that cluster-wide actual CPU usage and unit usage can be accounted for. The global monitor itself retrieves unit-usage information from the Hadoop resource manager directly.

In this prototype, only CPU and disk IO usage are considered by the local controllers. Conceivably, some job mixes may exist for which other types of resource usage also need to be considered. While we believe that the HSA approach will remain valid, we leave the detailed study and evaluation of these cases for future work and prototype extensions. In this prototype, THRESHOLD $C P U$ is set to $100 \%$, aiming at full CPU utilization. Once an update of MaxNumUnits is determined, the local controllers individually call a Hadoop command, yarn rmadmin -updateNodeResource, with updated value of MaxNumUnits. To avoid performance degradation because of disk IO contention, LocalIOUsage in the slave node is compared with ClusterIOUsage when updating MaxNumUnits. Then, running CPU-intensive jobs shows very low LocalIOUsage in slave nodes and so low ClusterIOUsage. In that case, it may occur that MaxNumUnits decreases even though it should increase because this is not the case of disk IO contention. A general method to find the appropriate value of disk IO in a node is beyond this article's scope. Instead, in this prototype implementation, disk IO contention is assumed when ClusterIOUsage is over $30 \%$, a threshold experimentally chosen from the static optimal case of the 500-TS sequence.

Hadoop provides the Capacity Scheduler and the Fair Scheduler, and both support to update configuration parameters for MaxNumJobs at runtime. The Capacity Scheduler's new configuration becomes effective immediately when the API call yarn rmadmin-refreshQueues is executed. For the Fair Scheduler, it takes too long (10-15 seconds) for the parameter update to become effective even after the scheduler notices that the parameters were modified [19]. Therefore, in this prototype implementation, the global controller interacts with the Hadoop Capacity Scheduler.

\section{EXPERIMENTAL EVALUATION}

\subsection{Experimental Setup}

The HSA is evaluated by using the prototype implementation on a 31-node (1 master and 30 slaves) cluster deployed on CloudLab. The cluster uses physical nodes on the m510 CloudLab cluster. Each node has an 8-core (16 threads) Intel ${ }^{\circledR}$ Xeon ${ }^{\circledR}$ CPU D-5540 2.0GHz, 64GB ECC Memory $(4 \times 16 \mathrm{~GB}$ DDR4-2133 SO-DIMMs), and 213GB assigned out of 512GB NVMe (non-volatile memory express) flash storage, running Ubuntu 16.04 LTS (Linux kernel 4.4.0-57-generic \#78-Ubuntu SMP x86_64). Apache $^{\mathrm{TM}}$ Hadoop ${ }^{\circledR}$ release 3.0.0-alpha2 (25 January 2017) is installed on the cluster. The master node runs the ResourceManager for YARN and NameNode for HDFS. Each slave node runs the NM for YARN and DataNode for HDFS. The HDFS settings are the same as the 10-slave Hadoop cluster ( 3 of replication factor and $64 \mathrm{MB}$ of block size). All experiments use Hadoop-default configurations except for the AMRP when using the HSA. 


\subsection{Workloads}

To demonstrate that the proposed approach improves performance when all the jobs show the same resource usage pattern, three different jobs, Grep (CPU-intensive), Terasort (IO-intensive), and Wordcount (balanced CPU and IO usage) are selected in the Hadoop examples. As stated, only $\mathrm{CPU}$ and disk IO utilizations are considered as resource usage in these experiments. The 500-GR, 500-TS, and 500-WC are composed of 500 consecutive Grep, Terasort, and Wordcount jobs, and each job in the job sequence takes $3 \mathrm{~GB}, 2 \mathrm{~GB}$, and $1 \mathrm{~GB}$ of input data. The datasets are generated by RandomTextWriter for both Grep and Wordcount and Teragen for Terasort in Hadoop. Each job in the sequence has the same number of tasks and is composed of $14 \mathrm{~K}$ tasks in total.

For the experiments with realistic workloads, a 500-SWIM sequence is constructed, consisting of the biggest jobs among the jobs generated by SWIM. To generate realistic and representative workloads from historical workloads, SWIM synthesizes workloads by sampling historical Hadoop traces. Our experiments use one trace from a 600-node cluster at Facebook for 6 months between May 2009 and October 2009, containing about 1 million jobs. From the historical trace, SWIM synthesizes roughly $6 \mathrm{~K}$ jobs. SWIM adjusts the job's size (i.e., number of tasks) proportionally to the number of nodes. Among the $6 \mathrm{~K}$ jobs, the biggest 500 jobs are selected for this experiment. The 500-SWIM sequence consists of small jobs after large jobs, which is one of the worst possible job sequences to be tackled. Under the Capacity Scheduler, an equal-opportunity-based scheduler, when significantly large jobs are followed by relatively small jobs, the small jobs may have to wait until some of the preceding large jobs finish. This can increase makespan. The 500-SWIM sequence for this experimental evaluation has a total of $26 \mathrm{~K}$ tasks and each job size ranges from 3 tasks to 6,595 tasks.

The jobs are submitted to the queue every 2 seconds, but job submission pauses as needed to limit the number of jobs waiting in the queue (e.g., no more than 30). Under these conditions, jobs can be launched whenever there are available units.

\subsection{Metrics and Configuration Control Cases}

Experiments are conducted varying MaxNumUnits and AMRP, the Hadoop parameter that is directly related to MaxNumJobs (more exactly, AMRP is a percentage rather than an absolute value and MaxNumJobs $=$ number of slave nodes $\times$ MaxNumUnits $\times$ AMRP/2). The results using the HSA are compared to ones with both the default configuration (i.e., AMRP $=0.1$ and MaxNumUnits $=16$ ) and the static optimal configuration. For the static optimal configuration, the value of MaxNumUnits is searched in the set $\{14,15,16,17,18,19,20\}$. The optimal value of AMRP is searched in the set $\{0.1,0.2,0.3 \ldots 1.0\}$. Makespan, resource usage, and individual job execution time are the metrics considered for comparison.

The prototype implementation provides three dynamic configuration options: MaxNumJobscontrol only, MaxNumUnits-control only, and control of both MaxNumJobs and MaxNumUnits (called MaxNumJobs-control, MaxNumUnits-control, and HSA, respectively). Figure 11 shows the makespan of a 500-SWIM sequence for each of the three control options, varying MaxNumUnits. Experiments with MaxNumUnits-control only show the worst makespan among the three control options. In the prototype implementation, MaxNumJobs can increase as the number of total units available in the cluster increases. Unless MaxNumUnits increases significantly, expansion of MaxNumJobs is limited. The workloads used in these experiments require a significant increase of concurrent jobs, but MaxNumUnits-control does not satisfy the demand. On the other hand, the MaxNumJobs-control option shows slightly less performance than the HSA option with the given workload. However, with different jobs, particularly, long-running jobs each of which consist of a small number of tasks, the HSA surpasses the MaxNumJobs-control because when all the units 


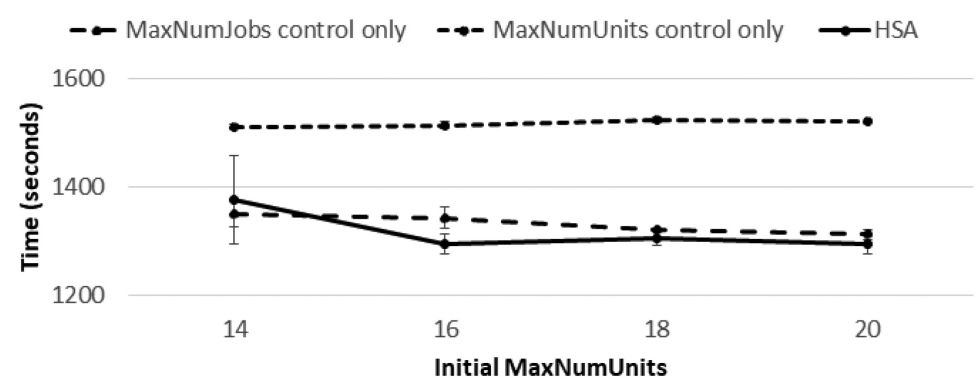

Fig. 11. Makespan of the 500-SWIM sequence for each one of the three configuration options: MaxNumJobscontrol only, MaxNumUnits-control only, and HSA as varying MaxNumUnits. The horizontal axis shows the value of MaxNumUnits at the beginning of workload execution. This value remains constant during workload execution in the case of MaxNumJobs-control only but varies for the other two cases.

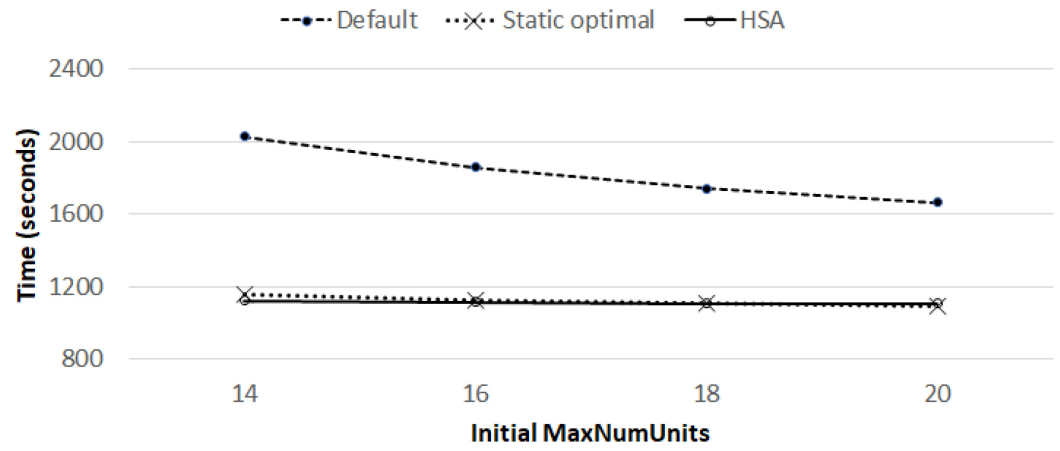

Fig. 12. Makespan of the 200-SWIM sequence in a 10-slave Hadoop cluster. "Default" and "static optimal" are for static configurations (i.e., the MaxNumUnits value during workload execution remains the same as its initial value). "HSA" is for the dynamic method. HSA's initial configurations are the same as the default case (AMRP $=0.1$ ), but MaxNumJobs and MaxNumUnits are updated on the fly. When MaxNumUnits is fixed to 14 , the best makespan of the static optimal was achieved when MaxNumJobs $=28$ $\left(10_{\text {nodes }} \times 14_{\text {MaxNumUnits }} \times 0.4_{\text {AMRP }} / 2\right) ;$ MaxNumJobs $=32\left(10_{\text {nodes }} \times 16_{\text {MaxNumUnits }} \times 0.4_{\mathrm{AMRP}} / 2\right)$ when MaxNumUnits $=16 ;$ MaxNumJobs $=45\left(10_{\text {nodes }} \times 18_{\text {MaxNumUnits }} \times 0.5_{\mathrm{AMRP}} / 2\right)$ when MaxNumUnits $=$ 18 ; and MaxNumJobs $=40\left(10_{\text {nodes }} \times 20_{\text {MaxNumUnits }} \times 0.4 \mathrm{AMRP} / 2\right) \mathrm{AMRP}=0.4$ when MaxNumUnits $=$ 20. MaxNumUnits for HSA indicates the initial value. Note that MaxNumJobs = number of slave nodes $\times$ MaxNumUnits $\times \mathrm{AMRP} / 2$.

are occupied by jobs and the maximum number of allowed jobs have been launched, increasing MaxNumUnits allows new jobs to be launched. Hence, in this article only the HSA control option is compared to the usage of the Capacity Scheduler with static values of MaxNumJobs and MaxNumUnits.

\subsection{Experimental Results}

A 200-SWIM job sequence was executed in the 10-slave Hadoop cluster. Figure 12 shows that the best makespan of the static optimal case does not change much even with different initial MaxNumUnits. When AMRP $=0.4$ and MaxNumUnits $=16,32$ jobs $\left(=16_{\text {MaxNumUnits }} \times 10_{\text {nodes }} \times 0.4_{\text {AMRP }} /\right.$ $\left.2_{\text {units per AM }}\right)$ can run simultaneously and a few changes to the number of jobs $(<10)$ do not affect the overall performance significantly. On the other hand, in the default case, when MaxNumUnits increases, the makespan is improved significantly because MaxNumJobs approaches the 


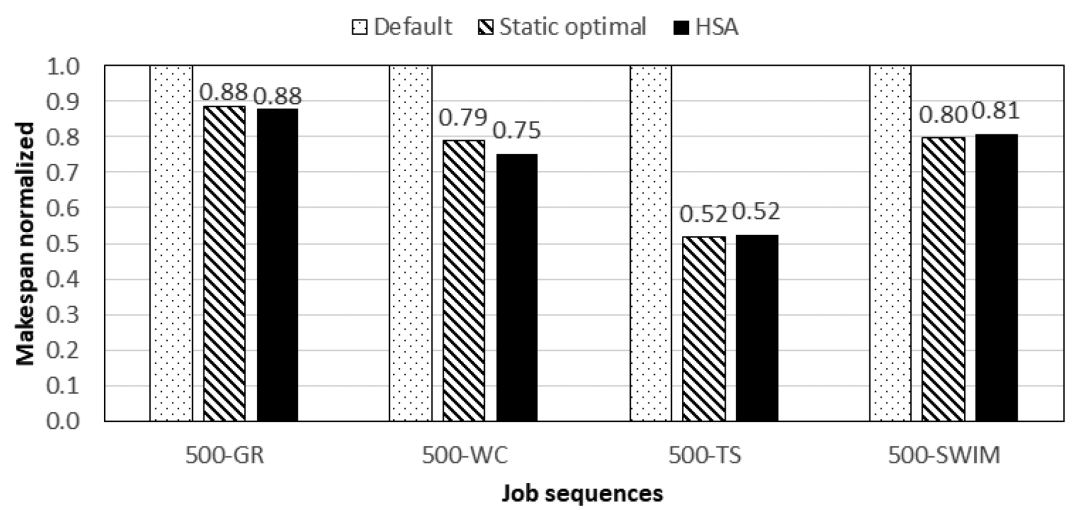

Fig. 13. Makespan of 500-job sequences. "Default" and "static optimal" results are for static configurations. "Default" results are achieved when AMRP $=0.1$ and MaxNumUnits $=16$. "Static optimal" results are found by exhaustive search. For the $500-$ GR sequence, the "static optimal" is achieved when MaxNumJobs $=81$ $\left(=30_{\text {nodes }} \times 18\right.$ MaxNumUnits $\left.\times 0.3_{\mathrm{AMRP}} / 2\right)$ and MaxNumUnits $=18$; for the 500-TS, when MaxNumJobs $=$ $81\left(=30_{\text {nodes }} \times 18_{\text {MaxNumUnits }} \times 0.3_{\mathrm{AMRP}} / 2\right)$ and MaxNumUnits $=18$; for the $500-\mathrm{WC}$, MaxNumJobs $=60$ $\left(=30_{\text {nodes }} \times 20_{\text {MaxNumUnits }} \times 0.2 \mathrm{AMRP} / 2\right)$ and MaxNumUnits $=20$; and for the 500-SWIM, MaxNumJobs $=90$ $\left(=30_{\text {nodes }} \times 20_{\text {MaxNumUnits }} \times 0.3_{\mathrm{AMRP}} / 2\right)$ and MaxNumUnits $=20$. HSA's initial configurations are the same as the "default" case $($ AMRP $=0.1$ and MaxNumUnits $=16)$.

static optimal case. The HSA starts with the default configurations AMRP $=0.1$, for different MaxNumUnits. As shown in Figure 12, the HSA presents similar makespan to the static optimal case regardless of the initial MaxNumUnits. That is, HSA can adjust MaxNumUnits and MaxNumJobs to achieve the static optimal performance. Makespan changes of the 500 -job sequences for different MaxNumUnits and MaxNumJobs show similar patterns to those observed for a 200-SWIM sequence in the 10-slave cluster, hence the best makespan of the static optimal cases are shown below only for the 500 -job sequences.

Figure 13 shows the normalized makespan of the four 500-job sequences: 500-GR, 500-TS, 500WC, and 500-SWIM. For our experimental setup the recommended MaxNumUnits is 16, following rules of thumb. For the static optimal cases, all the 500-job sequences show the best makespan with different MaxNumJobs and MaxNumUnits values from each other. For example, the 500-GR sequence shows its best makespan when MaxNumJobs $=81(\mathrm{AMRP}=0.3)$ and MaxNumUnits $=$ 18, the 500-TS sequence when MaxNumJobs $=81(\mathrm{AMRP}=0.3)$ and MaxNumUnits $=18$, the 500WC sequence when MaxNumJobs $=60($ AMRP $=0.2)$ and MaxNumUnits $=20$, and the 500-SWIM sequence when MaxNumJobs $=90(\mathrm{AMRP}=0.3)$ and MaxNumUnits $=20$. For all the experiments above with the 500-job sequences, HSA's makespan reduction over the default setup (with AMRP = 0.1 and MaxNumUnits = 16) matched the reduction achieved by the static optimal setup for the 200 -SWIM sequence (up to $42 \%$ ) and matched the reduction for the 500 -job sequences (up to $48 \%$ ). Each per-node local controller determines the appropriate number of MaxNumUnits based on CPU and disk IO utilization in the node. For all the 500-job sequences, their best makespan was achieved when MaxNumUnits is set to 18 or 20 over the default setup. This shows that the number of tasks that each node can tolerate (i.e., the CPU load) is as high as MaxNumUnits. Grep is a representative CPU-intensive job in Hadoop examples and its tasks consume CPU intensively. Hence, MaxNumUnits for the best makespan of the 500-GR sequence is less than for the other job sequences (i.e., 18 for the 500-GR sequence instead of 20). Though HSA starts with MaxNumUnits = 16 , it ends up with the optimal MaxNumUnits. 


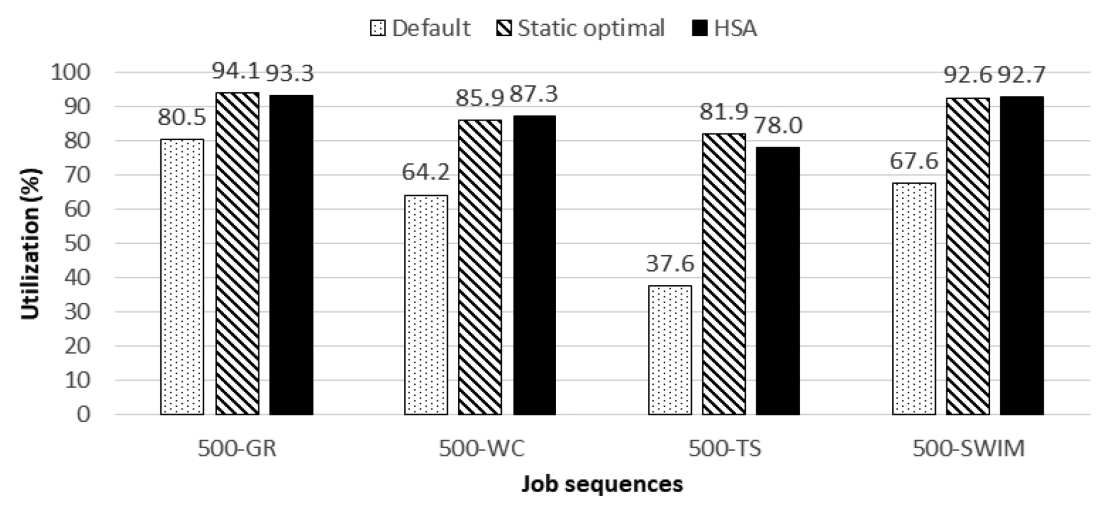

Fig. 14. Average CPU utilization while the 500-job sequences run. "Default" and "static optimal" results are for static configurations. "Default" results are achieved when AMRP $=0.1$ and MaxNumUnits $=16$. On the other hand, "Static optimal" results are found by exhaustive search. HSA's initial configurations are the same as the "default" case (AMRP = 0.1 and MaxNumUnits = 16), but MaxNumJobs and MaxNumUnits are dynamically updated at runtime.

Figure 14 presents average CPU utilization of the 500-job sequences' runs. Given that AMRP = 0.1 and MaxNumUnits $=16$ for the default configurations, the allowed number of concurrent jobs/tasks are not enough for full CPU utilization (i.e., the cluster is under-utilized). On the other hand, both the static optimal and the HSA improve CPU utilization significantly. The static optimal case can execute more jobs than the HSA initially because MaxNumJobs of the static optimal case is initialized to a higher value than the initial MaxNumJobs of HSA. HSA adjusts MaxNumJobs dynamically to execute as many jobs as it can, considering available resources. The improvement of CPU utilization of the 500-GR is relatively smaller than for the other 500-job sequences because Grep is CPU-intensive and the given workloads for the 500-GR sequence already consume about $80 \%$ of CPU even with the default configurations. Hence, there is not much room to improve CPU utilization for the 500-GR sequence in the given environment.

Figure 15 shows how many jobs run concurrently at a given time and how their number changes over time. For the default and static optimal cases, MaxNumJobs is static, so the maximum number of concurrent jobs is at most MaxNumJobs. On the other hand, HSA gradually increases MaxNumJobs based on CPU utilization in the Hadoop cluster (Figure 15(a) and (b)). Figure 15(c) shows the change in MaxNumJobs when running the 500-TS sequence. Because Terasort is IO-intensive and, as shown in Figure 14, CPU usage is low, the global controller just keeps increasing MaxNumJobs until ClusterIOUsage reaches the threshold value (i.e., 30\%). Given that Terasort is composed of fewer tasks than Grep and Wordcount in the experiments (see Table 1), more Terasort jobs can run simultaneously. Figure 15(d) shows the change in MaxNumJobs when running the 500-SWIM sequence. The 500-SWIM sequence was chosen from the biggest jobs in the SWIM jobs and sorted so that the earlier in the sequence a job is, the longer it is. The biggest SWIM jobs in the 500-SWIM sequence are big enough to occupy all units in the Hadoop cluster. Therefore, relatively small jobs just wait until the biggest SWIM jobs among the 500 jobs finish (576-second point) in the queue. Some small jobs consist of a small number of tasks, so it happens that there are many available units though the maximum number of small jobs are already running. Hence, HSA begins to increase MaxNumJobs in earnest from the 576-second point.

Figure 16 shows the number of units used by concurrent tasks (including AMs) during the 500 -job sequences run. The default and the static optimal cases limit the number of units because MaxNumUnits is fixed. On the other hand, the HSA adjusts MaxNumUnits as needed. Then, 


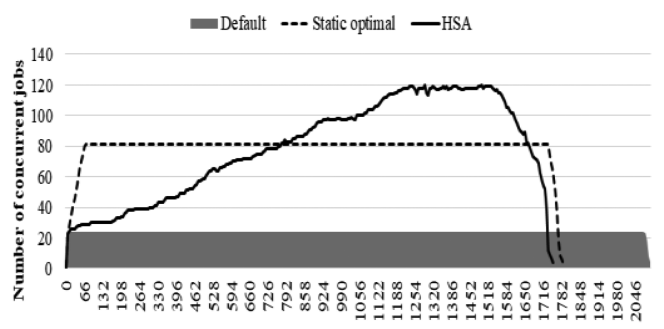

(a) 500-GR

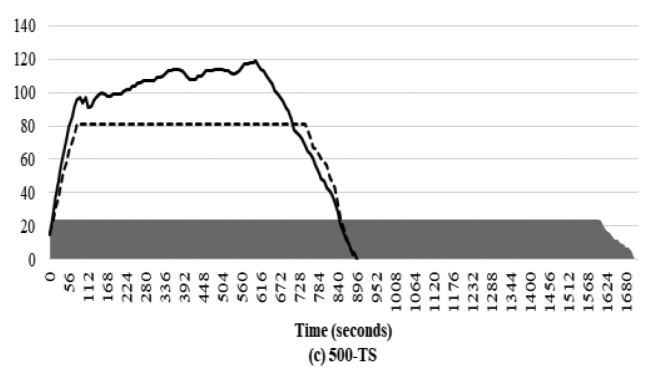

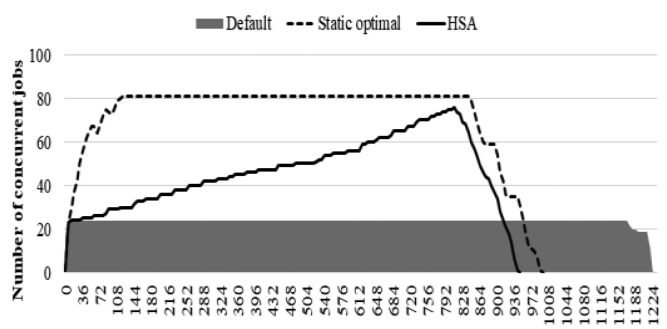

(b) 500-WC

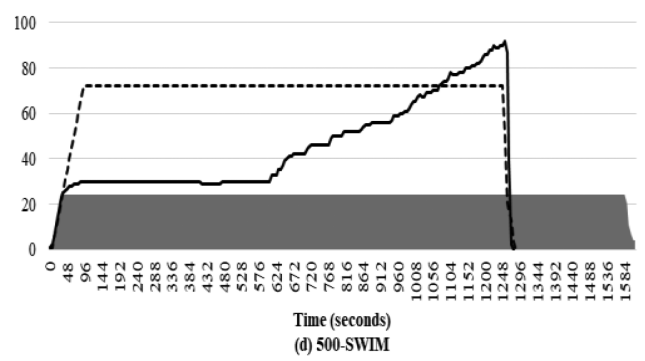

Fig. 15. Change of the number of concurrent jobs while the 500-job sequences run in the 30 -slave cluster.
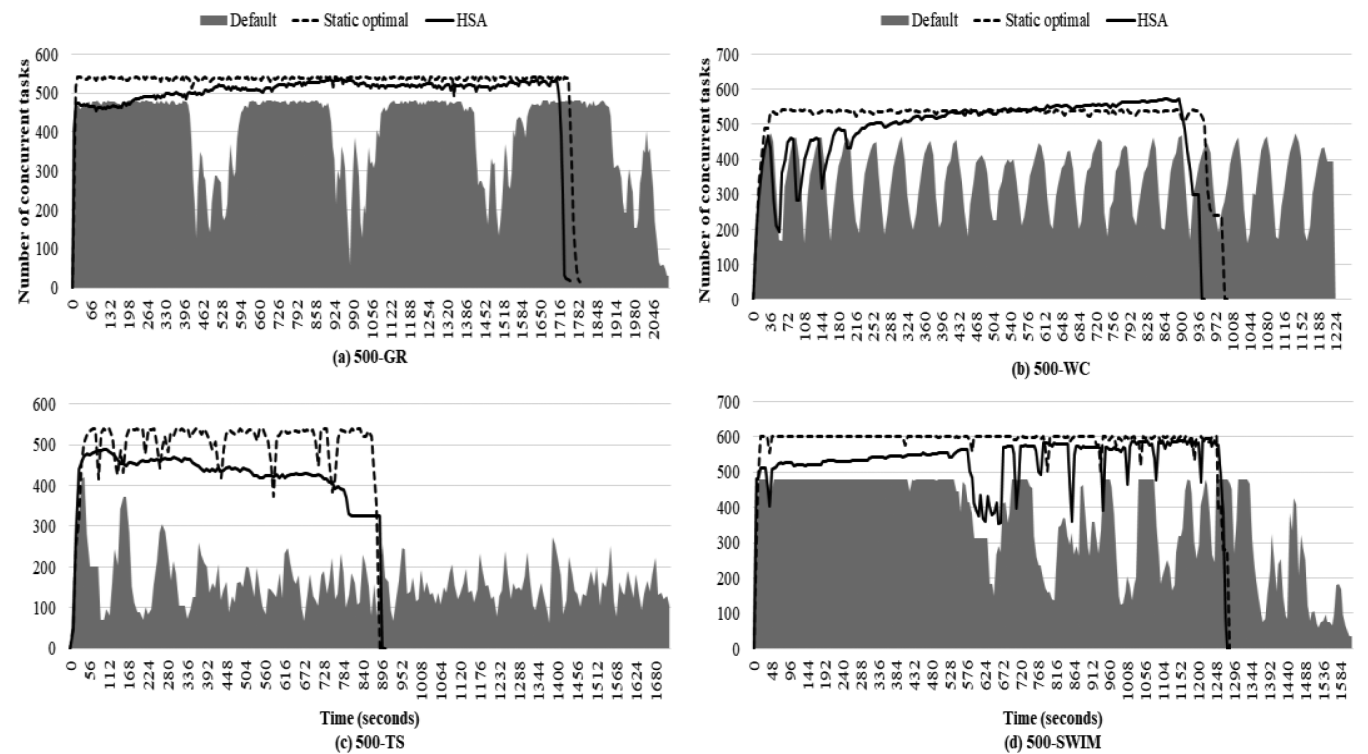

Fig. 16. The number of units used by concurrent tasks during the execution of each 500-job sequence when the control option is default, static optimal, and HSA. The total number of concurrent tasks includes twice the number of AMs because each AM uses two units.

Figure 16 shows deep drops of the number of concurrent tasks occasionally in the default cases of all the 500-job sequences. Given that the jobs in the 500-job sequences are composed of many mappers and relatively less reducers, at some point in time, resource units and CPU become under-utilized even though MaxNumJobs jobs run (when many of them are in the reduce phase). The default case may meet this condition with more probability than the other cases because MaxNumJobs of the default case is much smaller than for the other cases. In particular, Figure 16(c) 


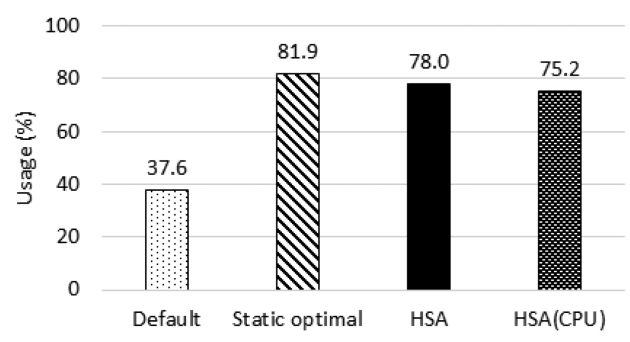

(a) average CPU usage

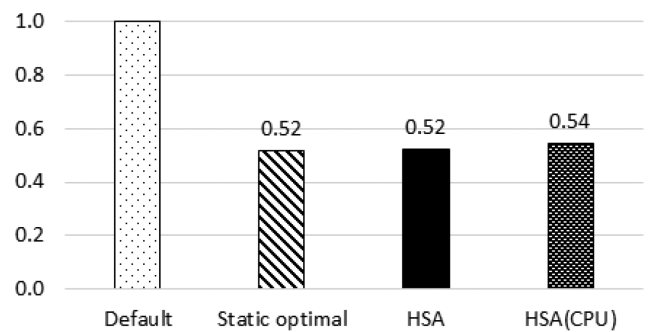

(b) normalized Makespan

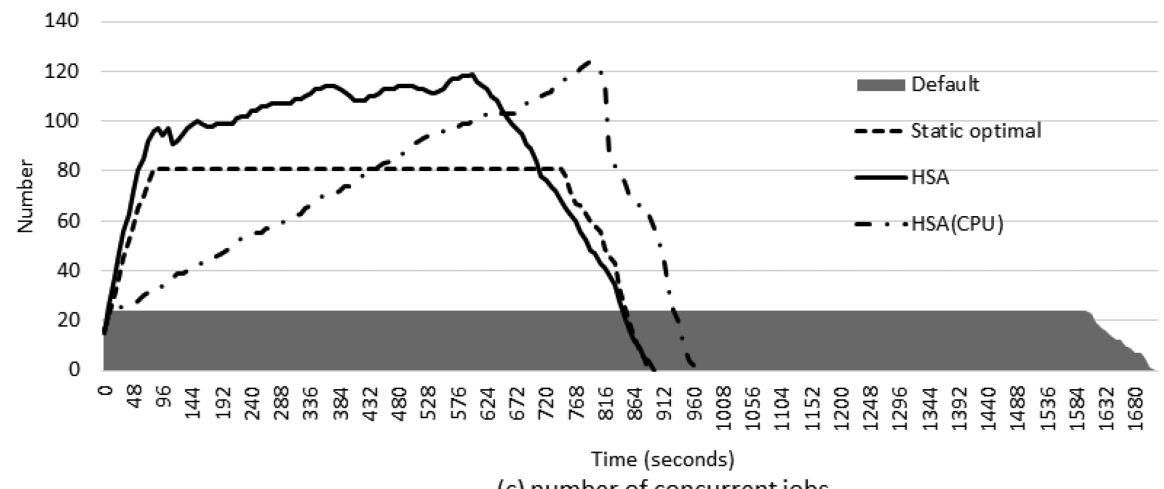

(c) number of concurrent jobs

Fig. 17. Comparison between HSA considering usage of CPU and disk IO (HSA) and HSA considering only CPU usage (HSA(CPU)) for the 500-TS sequence of jobs.

shows that the number of concurrent tasks keeps decreasing. Terasort in this experiment has one reducer which is time-consuming. That is, as time goes, many jobs of the 500-TS sequence run using only three units: two for AM and one for reduce task. Moreover, the reduce phase is IOintensive, so MaxNumJobs does not increase once ClusterIOUsage reaches the threshold values.

Taking into consideration the usage of both CPU and disk IO yields better results than considering only CPU usage when IO-intensive jobs (e.g., Terasort) are executed. To demonstrate this, a comparison between HSA taking usage of both CPU and disk IO into consideration and HSA with only CPU usage, i.e., HSA(CPU), was done by running the 500-Terasort (500-TS) sequence for both cases. The results show that the consideration of IO usage in HSA helps to further improve CPU utilization (Figure 17(a)) and makespan (Figure 17(b)) beyond what is achieved using only CPUusage control. While the 500-TS sequence runs, it occurs frequently that CPU usage is less than the CPU threshold because of IO wait. HSA(CPU) considers this situation as CPU-underutilization and the controller keeps increasing MaxNumJobs (Figure 17(c)).

Figure 18 shows the $\mathrm{CDF}$ (cumulative distribution function) of execution time of individual jobs of the 500-job sequences. All the jobs in each 500-job sequence are identical except for the 500SWIM sequence. In that sense, Figure 18(a) shows that for the Hadoop-default case, the execution time of about $90 \%$ of jobs in the 500-GR sequence is between 150 and 200 seconds while for the static optimal case, it is between 300 and 400 seconds. That is, individual jobs take longer to execute with the static optimal configurations. Grep is CPU-intensive, so the results are expectable because the static optimal case launches more jobs (i.e., 81 jobs) than the Hadoop default (i.e., 24 jobs) and they must share limited CPU resources. On the other hand, the execution time of the individual jobs for the HSA case is widely distributed between 100 and 500 seconds. Only about $10 \%$ of jobs show as good response time as the Hadoop-default case. Theoretically, about 13 Grep instances can 

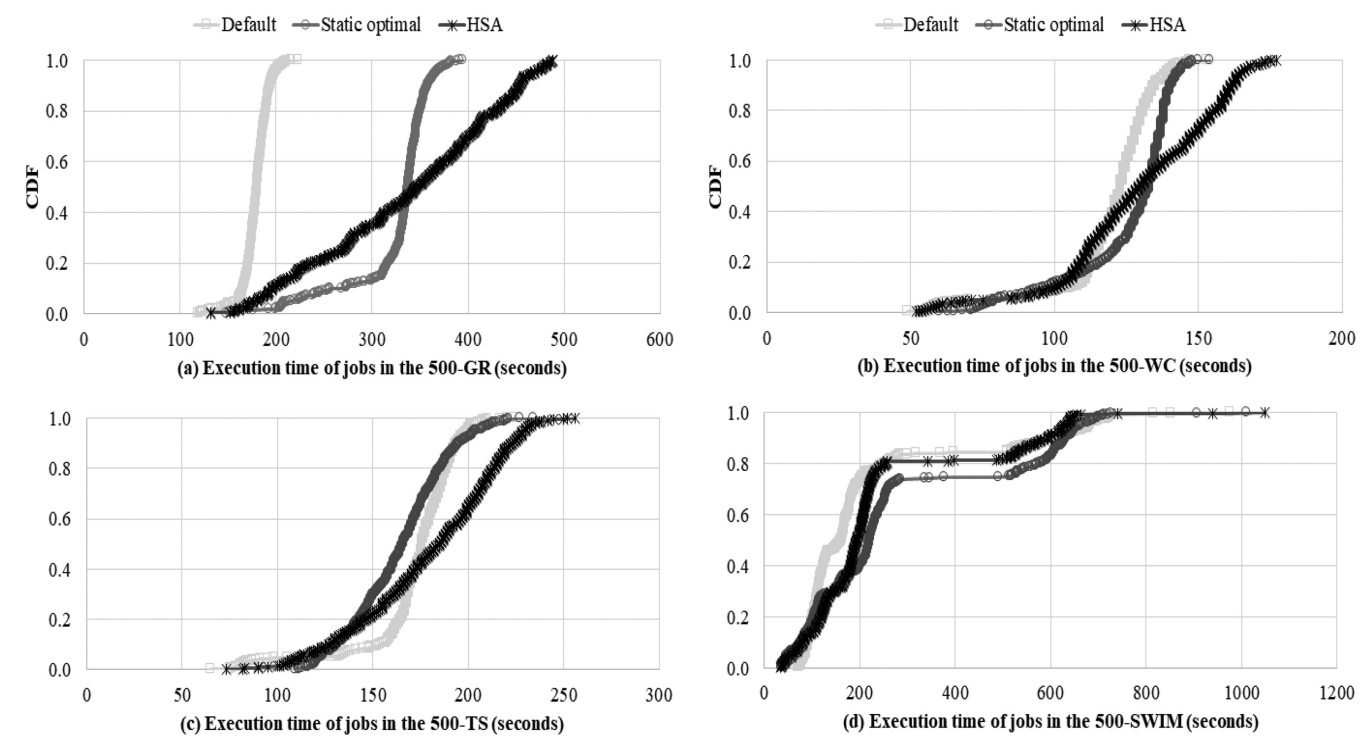

Fig. 18. CDF of each job execution time, running the 500-job sequences with MaxNumUnits $=16$. The job completion time includes wait time in the queue.

execute all the necessary map tasks at the same time in the given environment. As the HSA case is seeking optimal makespan, MaxNumJobs is increasing. That is, as time goes, more jobs run at the same time, sharing resources; they have longer execution time; and waiting time of jobs to be executed takes longer. Note that the execution time of a job includes the time it waits in the queue.

Figures 18(b) and (c) indicate that the execution time of individual jobs in both the 500-WC and 500-TS sequence has similar distribution regardless of the configuration options, showing that almost all jobs in each job sequence take longer than the shortest execution time. Some jobs under the HSA option show longer execution time than jobs under the other two options. The HSA begins with the Hadoop-default values and dynamically adjust MaxNumJobs and MaxNumUnits to seek the optimal value of them. In doing so, it only takes account of resource usage, but not the progress of individual jobs. This can end up with resource sharing by many jobs, so some jobs may take longer and wait longer in the queue.

As shown in Figure 18(d), the SWIM jobs can be roughly categorized into two groups: shortrunning group ( $<400$ seconds) and long-running group ( $>400$ seconds). Comparing to the default case, the HSA improves job completion time of the short-running group, but not so much for the long-running group. With respect to the jobs in the long-running group, they consume physical resources (i.e., CPU) fully. In other words, there is not much room to improve the job completion time of the biggest jobs because their resource utilizations are already near-optimal. In the static optimal case, some of long-running jobs take significantly longer than in the default case. The static optimal case occurs when AMRP $=0.3$. With MaxNumUnits $=20$, MaxNumJobs of the static optimal case is 90 jobs $\left(=30_{\text {nodes }} \times 20_{\text {MaxNumUnits }} \times 0.3_{\mathrm{AMRP}} / 2\right)$ while MaxNumJobs of the default case is $24\left(=30_{\text {nodes }} \times 16_{\text {MaxNumUnits }} \times 0.1_{\mathrm{AMRP}} / 2\right)$. The Capacity Scheduler keeps launching jobs in the queue as long as there are free units, limited by AMRP. Under the static optimal condition, more jobs in the long-running group are likely to be launched and some of those to use less resources than when they run under the default condition. This resource sharing pattern causes some jobs in the static optimal case to take longer than in the default case. Though overall makespan of the static optimal case shows the best performance, simply increasing MaxNumJobs can hurt individual 
job completion time. For the 500-SWIM sequence, the HSA can achieve improvement on jobs' completion time without hurting the jobs in the long-running group.

\section{RELATED WORK}

Self-tuning approaches mainly focus on performance improvement of individual jobs. To find better configurations, they construct performance models to predict future resource usage or job progress with current configurations and determine whether the current configurations are good or not. Having accurate performance models is critical for such approaches. Many techniques have been applied to construct such models [6, 7, 9, 11, 14, 22, 27, 28]. These model-based tuning approaches need model-training phases to achieve accurate prediction. Training is time-consuming and re-training or model re-construction are needed when input datasets or running environments are changed. The HSA is not model-based, so a time-consuming training phase is not needed. These model-based approaches focus on individual job's performance improvement, but our approach focuses on aggregate job performance (i.e., makespan) by improving resource utilization.

To address the limitations of model-based methods, online approaches have been proposed. While jobs are running, these approaches monitor resource usage and progress of individual tasks. Based on the monitored data, they try to find optimal configurations for tasks and apply the new configurations to future task waves. They seek to improve performance by maximizing the performance of individual tasks in each job $[2,4,13]$. Our approach seeks to maximize resource utilization in every node but does not focus on parameter configurations of individual tasks.

There are resource-usage-based approaches to control job concurrency $[12,30]$ and task concurrency [10]. They seek to avoid over-/under-utilization of resources in a cluster. [10] adjusts dynamically the total number of units according to resource usage in Hadoop. However, it requires modifications to Hadoop components. Moreover, it focuses on self-tuning for a single job run whereas the HSA considers multi-tenant environments. [12] uses a fuzzy-prediction approach, which generates and updates fuzzy rules on the fly. Hence, it can work appropriately when there is no a priori job's information. However, as dynamics of jobs increase, existing history-based rules need to be updated with current dynamics. Our approach has similar objectives to these resourceusage-based approaches. Instead of using historical data to update rules, the HSA uses rules based on the observation that CPU usage is highly correlated with the number of concurrent jobs. The HSA does not need rule updates.

Tasks in Hadoop 1 are assigned exclusively to corresponding task-specific units (i.e., map unit and reduce unit). The basic compute tasks are map and reduce and the number of units is statically determined. Hadoop 1 constrains map tasks to be executed prior to reduce tasks and map tasks and reduce tasks to be allocated to map units and reduce units, respectively. These constraints may cause low resource utilization in a Hadoop cluster, and so there are lots of studies to address this limitation by using dynamic unit allocation techniques [5, 15, 20, 21, 25]. Our approach uses dynamic adjustment of MaxNumUnits to improve performance by maximizing resource utilization in a node; furthermore, it is applied to Hadoop 3.

\section{CONCLUSIONS}

In this article, we describe a HSA to improve performance in data-analytics frameworks. It seeks performance improvement by maximizing resource utilization. In the proposed architecture, the per-cluster global controller controls MaxNumJobs using a fuzzy-logic based controller. The per-node local controllers adjust MaxNumUnits in each node based on the observed resource utilization. Each local controller executes its control operations by itself, but the global controller manages their control actions by checking out unit-usage information from the data-analytics framework and the local monitors. The proposed architecture was implemented in Hadoop 3. The 
prototype implementation demonstrated that dynamic adjustment of MaxNumJobs and MaxNumUnits based on CPU and disk IO usage improves both unit utilization and physical-resource utilization. This resulted in significant improvement of makespan and job completion time compared to Hadoop-default configurations. The achieved performance is similar to that obtained by optimal static configurations which can only be devised by exhaustive search and experimentation. Furthermore, no modifications to applications and frameworks are required, except for dynamically adjusting two configuration parameters (MaxNumJobs and MaxNumUnits in the case of the prototype implementation in Hadoop 3).

Regarding future work, the local monitor in the prototype monitors both the resource usage (i.e., CPU and disk IO) and the capacity in a slave node and uses the obtained information to avoid contention among tasks. However, just considering such information may be insufficient to further improve the response time of individual jobs in data-analytics framework. Therefore, a study to understand the correlation between job/task concurrency and makespan and response time of individual jobs may help devise adaptations that further improve overall performance. In addition, the proposed approach focuses on avoiding possible contention among tasks, adjusting MaxNumJobs and MaxNumUnits when contention happens and then waiting for the condition to be resolved. More active ways (e.g., job/task preemption) might be more effective and can be added in the proposed approach.

\section{ACKNOWLEDGMENTS}

The authors would like to thank the reviewers for their valuable comments which greatly improved the quality of the article.

\section{REFERENCES}

[1] Yanpei Chen, Archana Ganapathi, Rean Griffith, and Randy Katz. 2011. The case for evaluating mapreduce performance using workload suites. In Proceedings of the 2011 IEEE 19th International Symposium on Modeling, Analysis \& Simulation of Computer and Telecommunication Systems (MASCOTS). IEEE, 390-399.

[2] Dazhao Cheng, Jia Rao, Yanfei Guo, and Xiaobo Zhou. 2014. Improving mapreduce performance in heterogeneous environments with adaptive task tuning. In Proceedings of the 15th International Middleware Conference. ACM, 97-108.

[3] Jeffrey Dean and Sanjay Ghemawat. 2008. Mapreduce: Simplified data processing on large clusters. Communications of the ACM 51, 1 (2008), 107-113.

[4] Xiaoan Ding, Yi Liu, and Depei Qian. 2015. Jellyfish: Online performance tuning with adaptive configuration and elastic container in Hadoop yarn. In Proceedings of the 2015 IEEE 21st International Conference on Parallel and Distributed Systems (ICPADS'15). IEEE, 831-836.

[5] Zhenhua Guo, Geoffrey Fox, Mo Zhou, and Yang Ruan. 2012. Improving resource utilization in mapreduce. In Proceedings of the 2012 IEEE International Conference on Cluster Computing (CLUSTER'12). IEEE, 402-410.

[6] Mohammad Hammoud and Majd F. Sakr. 2013. Mc2: Map concurrency characterization for mapreduce on the cloud. In Proceedings of the 2013 IEEE 6th International Conference on Cloud Computing (CLOUD'13). IEEE, 17-26.

[7] Herodotos Herodotou, Harold Lim, Gang Luo, Nedyalko Borisov, Liang Dong, Fatma Bilgen Cetin, and Shivnath Babu. 2011. Starfish: A self-tuning system for big data analytics. In CIDR. 261-272.

[8] Impetus. 2009. Advanced Hadoop Tuning and Optimizations. Retrieved on January 26, 2019 from http://www. slideshare.net/impetusinfo/ppt-on-advancedhadoop-tuning-n-optimisation.

[9] Selvi Kadirvel and José A. B. Fortes. 2012. Grey-box approach for performance prediction in map-reduce based platforms. In Proceedings of the 2012 21st International Conference on Computer Communications and Networks (ICCCN'12). IEEE, 1-9.

[10] Kamal Kc and Vincent W. Freeh. 2015. Dynamically controlling node-level parallelism in Hadoop. In Proceedings of the 2015 IEEE 8th International Conference on Cloud Computing (CLOUD'15). IEEE, 309-316.

[11] Palden Lama and Xiaobo Zhou. 2012. Aroma: Automated resource allocation and configuration of mapreduce environment in the cloud. In Proceedings of the 9th International Conference on Autonomic Computing. ACM, 63-72.

[12] Gil Jae Lee and José A. B. Fortes. 2016. Hadoop performance self-tuning using a fuzzy-prediction approach. In Proceedings of the 2016 IEEE International Conference on Autonomic Computing (ICAC'16). IEEE, 55-64. 
[13] Min Li, Liangzhao Zeng, Shicong Meng, Jian Tan, Li Zhang, Ali R. Butt, and Nicholas Fuller. 2014. Mronline: Mapreduce online performance tuning. In Proceedings of the 23rd International Symposium on High-Performance Parallel and Distributed Computing. ACM, 165-176.

[14] Guangdeng Liao, Kushal Datta, and Theodore L. Willke. 2013. Gunther: Search-based auto-tuning of mapreduce. In Proceedings of the Euro-Par 2013 Parallel Processing. Springer, 406-419.

[15] Jorda Polo, David Carrera, Yolanda Becerra, Malgorzata Steinder, and Ian Whalley. 2010. Performance-driven task co-scheduling for mapreduce environments. In Proceedings of Network Operations and Management Symposium (NOMS'10). IEEE, 373-380.

[16] Albert Reuther, Chansup Byun, William Arcand, David Bestor, Bill Bergeron, Matthew Hubbell, Michael Jones, Peter Michaleas, Andrew Prout, Antonio Rosa, and Jeremy Kepner. 2016. Scheduler technologies in support of high performance data analysis. In Proceedings of the High Performance Extreme Computing Conference (HPEC'16). IEEE, $1-6$.

[17] Robert Ricci, Eric Eide, and Cloud Lab Team. 2014. Introducing CloudLab: Scientific infrastructure for advancing cloud architectures and applications. USENIX \& SAGE 39, 6 (2014), 36-38.

[18] Hadoop: Capacity Scheduler. Retrieved from http://hadoop.apache.org/docs/current/hadoop-yarn/hadoop-yarn-site/ capacityscheduler.html.

[19] Hadoop: Fair Scheduler. Retrieved from http://hadoop.apache.org/docs/current/hadoop-yarn/hadoop-yarn-site/ fairscheduler.html.

[20] Bikash Sharma, Ramya Prabhakar, S. Lim, Mahmut T. Kandemir, and Chita R. Das. 2012. Mrorchestrator: A FineGrained Resource Orchestration Framework for Hadoop Mapreduce. Technical Report CSE-12-001, Pennsylvania State University.

[21] Shanjiang Tang, Bu-Sung Lee, and Bingsheng He. 2014. Dynamicmr: A dynamic slot allocation optimization framework for mapreduce clusters. IEEE Transactions on Cloud Computing 2, 3 (2014), 333-347.

[22] Fengguang Tian and Keke Chen. 2011. Towards optimal resource provisioning for running mapreduce programs in public clouds. In Proceedings of the 2011 IEEE International Conference on Cloud Computing (CLOUD'11). IEEE, 155-162.

[23] Werner Van Leekwijck and Etienne E. Kerre. 1999. Defuzzification: Criteria and classification. Fuzzy Sets and Systems 108, 2 (1999), 159-178.

[24] Vinod Kumar Vavilapalli, Arun C. Murthy, Chris Douglas, Sharad Agarwal, Mahadev Konar, Robert Evans, Thomas Graves, Jason Lowe, Hitesh Shah, Siddharth Seth, Bikas Saha, Carlo Curino, Owen O’Malley, Sanjay Radia, Benjamin Reed, and Eric Baldeschwieler. 2013. Apache Hadoop yarn: Yet another resource negotiator. In Proceedings of the 4th Annual Symposium on Cloud Computing. ACM, 5.

[25] Abhishek Verma, Ludmila Cherkasova, and Roy H. Campbell. 2011. Aria: Automatic resource inference and allocation for mapreduce environments. In Proceedings of the 8th ACM International Conference on Autonomic Computing. ACM, 235-244.

[26] Tom White. 2012. Hadoop: The Definitive Guide. O’Reilly Media, Inc.

[27] Nezih Yigitbasi, Theodore L. Willke, Guangdeng Liao, and Dick Epema. 2013. Towards machine learning-based autotuning of mapreduce. In Proceedings of the 2013 IEEE 21st International Symposium on Modeling, Analysis \& Simulation of Computer and Telecommunication Systems (MASCOTS'13). IEEE, 11-20.

[28] Zhibin Yu, Zhendong Bei, Huiling Zhang, Wen Xiong, Lieven Eeckhout, Chengzhong Xu, and Shenzhong Feng. 2016. RFHOC: A random-forest approach to auto-tuning Hadoop's configuration. IEEE Transactions on Parallel and Distributed Systems 27, 5 (2016), 1470-1483.

[29] Lotfi A. Zadeh. 1965. Fuzzy sets. Information and control 8, 3 (1965), 338-353.

[30] Bo Zhang, F. Krikava, R. Rouvoy, and L. Seinturier. 2015. Self-configuration of the number of concurrently running mapreduce jobs in a Hadoop cluster. In Proceedings of the 2015 IEEE International Conference on Autonomic Computing (ICAC'15). 149-150.

[31] F. Yan, L. Cherkasova, Z. Zhang, and E. Smirni. 2017. Dyscale: A mapreduce job scheduler for heterogeneous multicore processors. IEEE Transactions on Cloud Computing 5, 2 (2017), 317-330.

Received February 2018; revised December 2018; accepted January 2019 